\title{
Trends and determinants of undernutrition among young Kenyan children: Kenya Demographic and Health Survey; 1993, 1998, 2003 and 2008-2009
}

\author{
Peninah K Masibo* and Donald Makoka \\ Department of Epidemiology and Nutrition, School of Public Health, Moi University, Nairobi Campus, PO Box \\ 63056 - 00200, Nairobi, Kenya
}

Submitted 17 January 2012: Final revision received 30 April 2012: Accepted 4 May 2012: First published online 14 June 2012

\begin{abstract}
Objective: To report on the trends and determinants of undernutrition among children $<5$ years old in Kenya.

Design: Data from four nationwide Kenya Demographic and Health Surveys, conducted in 1993, 1998, 2003 and 2008-2009, were analysed. The Demographic and Health Survey utilizes a multistage stratified sampling technique.

Setting: Nationwide covering rural and urban areas in Kenya.

Subjects: The analysis included 4757, 4433, 4892 and 4958 Kenyan children aged $<5$ years in 1993, 1998, 2003 and 2009-2009, respectively.

Results: The prevalence of stunting decreased by 4.6 percentage points from $39.9 \%$ in 1993 to $35.3 \%$ in 2008-2009, while underweight decreased by $2 \cdot 7$ percentage points from $18.7 \%$ in 1993 to $16.0 \%$ in $2008-2009$. The effects of household wealth, maternal education and current maternal nutritional status on child nutrition outcomes have changed dynamically in more recent years in Kenya. Inadequate hygiene facilities increased the likelihood of chronic undernutrition in at least three of the surveys. Small size of the child at birth, childhood diarrhoea and male gender increased the likelihood of undernutrition in at least three of the surveys. Childhood undernutrition occurred concurrently with maternal overnutrition in some households.

Conclusions: The analysis reveals a slow decline of undernutrition among young children in Kenya over the last three decades. However, stunting and underweight still remain of public health significance. There is evidence of an emerging trend of a malnutrition double burden demonstrated by stunted and underweight children whose mothers are overweight.
\end{abstract}

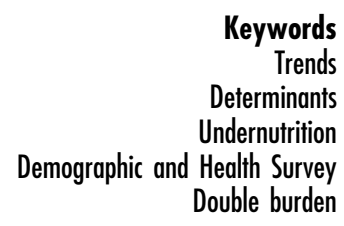

eywords Determinants Undernutrition Double burden
The importance of child nutritional status as an indicator for tracking the nutrition and health status of populations is well recognized ${ }^{(1)}$. Malnutrition leads to a reduction of human performance, deterioration of health and reduced survival of populations ${ }^{(2,3)}$, as well as hindering the potential for countries to reduce poverty and maximize socio-economic development ${ }^{(4)}$. Childhood undernutrition negatively affects school performance, thus lowering adult incomes, which in turn has negative implications for national development ${ }^{(5)}$. The link between malnutrition, morbidity and child mortality makes undernutrition the underlying cause of over half of all child deaths ${ }^{(6)}$. This is especially so in the Sub-Saharan Africa region, where it is estimated that $4 \cdot 8$ million children die before the age of 5 years each year ${ }^{(7)}$.

Child undernutrition is one of Africa's most fundamental challenges for improved human development, thus slowing down achievement of the goal of reducing child malnutrition in most developing countries ${ }^{(8)}$. The prevalence of childhood undernutrition has remained of public health significance in Kenya in spite of efforts by government and non-government agencies to deal with the problem ${ }^{(9)}$. The high rates of child undernutrition together with high rates of child mortality continue to deter the government's efforts to provide quality health care towards the reduction of mortality and malnutrition levels ${ }^{(10)}$. While studies conducted previously have identified the magnitude and determinants of undernutrition in Kenya and other low-income settings ${ }^{(10,11)}$, few studies have examined the trends of undernutrition and how the effect of identified determinants on child nutritional status changes over time. The current analysis describes the trends of chronic and acute malnutrition among Kenyan children over a period of two-and-half decades and examines the changing patterns of the effect of selected household, maternal and child-related 
characteristics on child nutritional status based on data from four Demographic and Health Surveys (DHS).

\section{Methods}

The present analysis utilized data from the nationwide Kenya Demographic and Health Surveys (KDHS) conducted from 1993 to 2008-2009. A total of 8805 households were selected for inclusion in 1993, 9465 in 1998, 9865 in 2003 and 9936 in 2008-2009, with a household response rate of $96.3 \%$ or above in all of the surveys. The 1993 and 1998 samples excluded the North Eastern Province and four other northern districts (Samburu and Turkana in Rift Valley Province; Isiolo and Marsabit in Eastern Province) because of security reasons. The excluded districts accounted for less than $4 \%$ of Kenya's population.

The DHS utilizes a multistage stratified cluster sampling methodology in which samples of households within clusters (enumeration areas) are selected. Households are then systematically selected within each cluster and household residents are eligible to participate in the survey. Urban areas are oversampled and the present analysis was based on weighted data to cater for the different sample proportions. The analysis included children $<5$ years of age whose weight and height/length measurements were taken and whose mothers were interviewed and had their weights and heights measured.

\section{Anthropometric measurements}

Weights and heights were taken for all children aged $<5$ years and their mothers in participating households. Measurements were taken with participants wearing light clothing without shoes or socks. Weight was measured to the nearest $0 \cdot 1 \mathrm{~kg}$ using a light bathroom-type scales, while length (children aged $<24$ months) or height (children aged $\geq 24$ months) was measured to the nearest $0 \cdot 1 \mathrm{~cm}$ using calibrated height boards ${ }^{(12)}$. Undernutrition was determined as stunting, wasting and underweight defined by the WHO 2006 growth reference standards, which use the WHO Multicentre Growth Reference Study population. Stunting, wasting and underweight were defined as height/length-for-age, weight-for-height/length and weight-for-age less than $-2 \mathrm{SD}$ (Z-score) from the reference standards for children of the same age and sex, respectively ${ }^{(13)}$. Weight-for-height/length $Z$-score was calculated for those with heights of 65 to $120 \mathrm{~cm}$.

\section{Background characteristics}

Background characteristics included in the present analysis were urban or rural residence, province, gender of the household head, wealth index, source of drinking water, and availability and type of toilet facility.

\section{Wealth index}

Household wealth index is a socio-economic index constructed as an indicator of the level of wealth that is consistent with expenditure and income measures. In the DHS this is based on data about household ownership of assets and consumer goods such as source of drinking water, type of toilet facilities, type of fuel, ownership of various durable goods and other characteristics relating to socio-economic status of the household. A factor score generated through principal components analysis is assigned to each asset, and the resulting asset scores are standardized in relation to a normal distribution ${ }^{(14)}$. Each household is then assigned a score for each asset and the scores are summed for each household ${ }^{(15)}$. The proxy indicator for the long-term standard of living of the household is based on this score. For the present analysis the wealth index was grouped into five categories, namely poorest, poorer, middle, richer and richest.

\section{Housebold bygiene facilities}

Household hygiene facilities included were the source of drinking water and availability and type of toilet facility. Improved sources of drinking water included piped water, tap water, bottled water and protected wells in the compound. Unprotected wells, springs, rivers, ponds, lakes and dams were grouped as non-improved water sources. Improved household toilet facilities included flush toilets and ventilated improved pit (VIP) latrines, while traditional pit latrines were categorized as nonimproved household toilet facilities.

\section{Maternal characteristics}

Maternal characteristics included in the analysis were age, marital status, highest level of education obtained, BMI, work status and number of children $<5$ years of age living with the mother. Maternal BMI was used as a measure of the mother's current nutritional status at the time the child was measured. Maternal BMI was computed as weight in kilograms divided by the square of height in metres. BMI cutoffs were based on the recommended international cut-offs as follows: underweight was defined as BMI $<18.5 \mathrm{~kg} / \mathrm{m}^{2}$, normal weight as BMI $=18 \cdot 5-24.9 \mathrm{~kg} / \mathrm{m}^{2}$, overweight and obesity as $\mathrm{BMI} \geq 25 \cdot 0 \mathrm{~kg} / \mathrm{m}^{2}$. Pregnant mothers and those with babies two months postpartum were included in a separate category.

\section{Child characteristics}

Child characteristics included the child's age, gender, birth order, size at birth, and whether or not the child had diarrhoea, fever or cough in the last two weeks before the survey. Information on the child's relative size at birth as reported by the mother was grouped as small, average and large.

\section{Data analysis}

Data were analysed using the SPSS statistical software package version $18 \cdot 0$. Analysis was done at the descriptive, univariate, bivariate and multivariate levels. Pearson's $\chi^{2}$ test was used to check for associations between household, 
maternal and child-related characteristics and child undernutrition status in the four surveys at the bivariate level. Binary logistic regression was used to examine associations between the explanatory variables and the undernutrition outcomes: stunting, wasting and underweight. The adjusted regression model included household characteristics (namely residence, wealth index, province, source of drinking water and toilet facilities), maternal characteristics (namely age, marital status, education, BMI, work status and number of children $<5$ years old living with the mother) and child-related characteristics (namely sex, age, birth order, size at birth and illnesses (diarrhoea, fever and cough)). The analysis on determinants was carried out for the four KDHS separately and then data were pooled for analysis of trends in prevalence. The analyses were done using complex samples analysis to adjust for the cluster sampling design used in the DHS.

\section{Results}

The current study included children aged 0-59 months with a mean age of 29.5 months in $1993,28.3$ months in 1998, $27 \cdot 8$ months in 2003 and 28.7 months in 2008-2009. The largest proportion of children was sampled from the Rift Valley Province $(21 \cdot 8 \%, 25 \cdot 7 \%, 26 \cdot 8 \%$ and $28 \cdot 3 \%$ in 1993, 1998, 2003 and 2008-2009, respectively; Table 1). On average, more than $80 \%$ of the children lived in rural areas compared with about $17 \%$ in urban areas. The mean height and weight of the children in all surveys was $82 \mathrm{~cm}$ and $11 \mathrm{~kg}$. There were no significant age differences noted by children's gender. The mean height-for-age $Z$-score increased slightly from -1.6 in 1993 to -1.4 in 2008-2009. The prevalence of stunting decreased by 4.6 percentage points from $39.9 \%$ in 1993 to $35.3 \%$ in 2008-2009 (Table 2). Underweight declined by $2 \cdot 7$ percentage points over the same period, while wasting levels did not change. A multivariate analysis of pooled data using the year of the survey as a variable and 1993 as the reference category showed a significant $(P<0 \cdot 005)$ decline in the odds of being stunted or underweight with time (Fig. 1). Children were less likely to be stunted in the 1998, 2003 and 2008-2009 surveys and less likely to be underweight in 2003 and 2008-2009 $(P<0 \cdot 05)$ compared with 1993 . The unadjusted odds of stunting were $0 \cdot 9,0 \cdot 8$ and $0 \cdot 8$ in 1998, 2003 and 2008-2009, respectively (Fig. 1). The unadjusted odds of underweight were 0.8 times lower in 2003 and 2008-2009 compared with 1993 (Fig. 1).

\section{Household characteristics}

Place of residence, province, wealth index, source of drinking water, and availability and type of toilet facility were significantly associated with undernutrition. The proportion of stunted, wasted and underweight children was higher in rural areas compared with urban areas in all surveys (Table 2). The prevalence of underweight decreased from $20 \%$ in 1993 to $17 \%$ in 2008-2009 among the rural areas, while this prevalence remained at $10 \%$ in the urban areas during all surveys. The slow decline in the prevalence of stunting at the national level was also evident in the regions except for Rift Valley Province, which showed an increasing trend in stunting from $35.0 \%$ in 1993 to $37 \cdot 8 \%$ in 2003 and then a slight decline to $35 \cdot 8 \%$ in $2008-2009$ (Table 2).

Undernutrition was negatively correlated with wealth index throughout the four surveys. Children in the poorest, poorer, middle and richer wealth quintiles had higher relative odds $(1.5$ times $)$ of stunting $(P<0.05)$ compared with those in the richest quintile in the earlier surveys (1993 and 1998; Table 3). This trend changed in the more recent surveys (2003 and 2008-2009) where only children from the poorest wealth index were significantly more likely $(P<0.005)$ to be stunted in 2003 (1.6 times) and 2008-2009 (2.1 times; Table 3) compared with those from the richest wealth quintile. Slightly more than half $(51 \%)$ of the children living in households without toilet facilities were stunted in 1993, reducing to $48 \%$ in $1998,41 \%$ in 1998 and $42 \%$ in 2008-2009 (Table 2). Children in households without a toilet facility and with a non-improved toilet facility were $2 \cdot 2$ times and 1.5 times significantly $(P<0.05)$ more likely to be stunted in 1993. The effect of this factor changed in 2003 and 2008-2009 whereby the increased odds of stunting remained significant $(P<0.005)$ among children in households with a non-improved toilet facility (Table 3 ). The association between wasting and availability and type of toilet facilities was significant in all four surveys (all $P<0 \cdot 05$ ). More than a quarter (28\%) of the children living in households without a toilet facility were underweight in 1993, 1998, 2008-2009 while a quarter (25\%) were underweight in 2003 (Table 2). Regional differentials showed that children were significantly less likely to be stunted in Nyanza, Rift Valley and Western provinces in 1993 and 2008-2009 compared with Nairobi Province (Table 3 ).

\section{Maternal characteristics}

Mother's education, current maternal nutritional status and the number of children $<5$ years old living with the mother had significant associations with undernutrition during the period under consideration. There was an increased likelihood of stunting, wasting and underweight if the child's mother had no education compared with secondary or higher levels. The proportion of stunted and underweight children among non-educated mothers declined significantly with time (all $P<0 \cdot 001$; Table 2). Children of uneducated mothers were more likely to be stunted: 1.6 times more in $1993,1 \cdot 7$ times more in 1998 and 1.7 times more in 2003 (Table 3). While the odds of child stunting were not significant in 2008-2009 among children of non-educated mothers, the odds were significant $(P<0 \cdot 05)$ among children whose 
Table 1 Distribution of household, maternal and child-related characteristics: Kenya Demographic and Health Survey; 1993, 1998, 2003 and 2008-2009

\begin{tabular}{|c|c|c|c|c|c|c|c|c|}
\hline & \multicolumn{2}{|c|}{$\begin{array}{c}1993 \\
(n 4757)\end{array}$} & \multicolumn{2}{|c|}{$\begin{array}{c}1998 \\
(n 4413)\end{array}$} & \multicolumn{2}{|c|}{$\begin{array}{c}2003 \\
(n 4893)\end{array}$} & \multicolumn{2}{|c|}{$\begin{array}{c}2008-2009 \\
(n \text { 4958) }\end{array}$} \\
\hline & $n$ & $\%$ & $n$ & $\%$ & $n$ & $\%$ & $n$ & $\%$ \\
\hline \multicolumn{9}{|l|}{ Household characteristics } \\
\hline \multicolumn{9}{|l|}{ Residence } \\
\hline Rural & 4222 & $88 \cdot 7$ & 3662 & $83 \cdot 0$ & 4039 & $82 \cdot 6$ & 4102 & $82 \cdot 0$ \\
\hline Urban & 536 & $11 \cdot 3$ & 751 & $17 \cdot 0$ & 853 & $17 \cdot 4$ & 856 & $17 \cdot 3$ \\
\hline \multicolumn{9}{|l|}{ Province } \\
\hline North Easternt & & & & & 112 & $2 \cdot 3$ & 122 & $2 \cdot 5$ \\
\hline Central & 599 & $12 \cdot 6$ & 414 & $9 \cdot 4$ & 556 & $11 \cdot 4$ & 403 & $8 \cdot 1$ \\
\hline Coast & 376 & $7 \cdot 9$ & 346 & $7 \cdot 8$ & 401 & $8 \cdot 2$ & 440 & 8.9 \\
\hline Eastern & 980 & $20 \cdot 6$ & 753 & $17 \cdot 1$ & 812 & $16 \cdot 6$ & 800 & $16 \cdot 1$ \\
\hline Nyanza & 773 & $16 \cdot 2$ & 906 & $20 \cdot 5$ & 747 & $15 \cdot 3$ & 918 & $18 \cdot 5$ \\
\hline Rift Valley & 1038 & $21 \cdot 8$ & 1134 & $25 \cdot 7$ & 1312 & $26 \cdot 8$ & 1404 & $28 \cdot 3$ \\
\hline Western & 828 & $17 \cdot 4$ & 604 & $13 \cdot 7$ & 644 & $13 \cdot 2$ & 617 & $12 \cdot 4$ \\
\hline Nairobi & 163 & $3 \cdot 4$ & 257 & $5 \cdot 8$ & 308 & $6 \cdot 3$ & 254 & $5 \cdot 1$ \\
\hline \multicolumn{9}{|l|}{ Wealth index $\ddagger$} \\
\hline Poorest & 633 & $13 \cdot 3$ & 1076 & $24 \cdot 4$ & 1197 & $24 \cdot 5$ & 1215 & $24 \cdot 5$ \\
\hline Poorer & 621 & $13 \cdot 0$ & 947 & $21 \cdot 5$ & 1061 & $21 \cdot 7$ & 1035 & $20 \cdot 9$ \\
\hline Middle & 596 & $12 \cdot 5$ & 844 & $19 \cdot 1$ & 953 & $19 \cdot 5$ & 946 & $19 \cdot 1$ \\
\hline Richer & 571 & $12 \cdot 0$ & 800 & $18 \cdot 1$ & 836 & $17 \cdot 1$ & 895 & $18 \cdot 0$ \\
\hline Richest & 544 & $11 \cdot 4$ & 746 & $16 \cdot 9$ & 846 & $17 \cdot 3$ & 867 & $17 \cdot 5$ \\
\hline \multicolumn{9}{|l|}{ Source of drinking water§, „\| } \\
\hline Non-improved & 3547 & $74 \cdot 6$ & 3264 & $73 \cdot 6$ & 3063 & $63 \cdot 0$ & 2164 & $43 \cdot 7$ \\
\hline Improved & 1178 & $24 \cdot 8$ & 1156 & $26 \cdot 1$ & 1721 & $35 \cdot 0$ & 2735 & $55 \cdot 2$ \\
\hline \multicolumn{9}{|l|}{ Toilet facilities } \\
\hline No toilet facility & 808 & $17 \cdot 0$ & 2970 & $67 \cdot 0$ & 1009 & $20 \cdot 6$ & 889 & $17 \cdot 9$ \\
\hline Improved toilet & 3349 & $70 \cdot 4$ & 620 & $14 \cdot 0$ & 3130 & $65 \cdot 4$ & 2062 & $41 \cdot 6$ \\
\hline Non-improved & 566 & $11 \cdot 9$ & 820 & $18 \cdot 5$ & 646 & $13 \cdot 2$ & 1948 & $39 \cdot 3$ \\
\hline \multicolumn{9}{|l|}{ Maternal characteristics } \\
\hline Mother's age (years) & & & & & & & & \\
\hline$<20$ & 259 & $5 \cdot 4$ & 278 & $6 \cdot 3$ & 310 & $6 \cdot 3$ & 245 & $4 \cdot 9$ \\
\hline $20-30$ & 2884 & $60 \cdot 6$ & 2727 & $61 \cdot 8$ & 2937 & $60 \cdot 0$ & 3096 & $62 \cdot 4$ \\
\hline$>30$ & 1614 & $33 \cdot 9$ & 1409 & $31 \cdot 9$ & 1645 & $33 \cdot 6$ & 1618 & $32 \cdot 6$ \\
\hline Marital status & & & & & & & & \\
\hline Single & 828 & $17 \cdot 4$ & 701 & $15 \cdot 9$ & 660 & $13 \cdot 5$ & 999 & $20 \cdot 2$ \\
\hline Married & 3929 & $82 \cdot 6$ & 3712 & $84 \cdot 1$ & 4233 & $86 \cdot 5$ & 3959 & $79 \cdot 8$ \\
\hline Education & & & & & & & & \\
\hline No education & 829 & $17 \cdot 4$ & 487 & $11 \cdot 0$ & 694 & $14 \cdot 2$ & 629 & $12 \cdot 7$ \\
\hline Primary & 2841 & $59 \cdot 7$ & 2774 & $62 \cdot 9$ & 3146 & $64 \cdot 3$ & 3177 & $64 \cdot 1$ \\
\hline Secondary+ & 1088 & $22 \cdot 9$ & 1152 & $26 \cdot 1$ & 1052 & $21 \cdot 5$ & 1152 & $23 \cdot 2$ \\
\hline BMI category & & & & & & & & \\
\hline Pregnant and postpartum & 647 & $13 \cdot 6$ & 587 & $13 \cdot 3$ & 673 & $13 \cdot 8$ & 617 & $12 \cdot 4$ \\
\hline Thin $\left(<18 \cdot 5 \mathrm{~kg} / \mathrm{m}^{2}\right)$ & 438 & $9 \cdot 2$ & 484 & $11 \cdot 0$ & 560 & $11 \cdot 5$ & 572 & $11 \cdot 5$ \\
\hline Normal $\left(18.5-24.9 \mathrm{~kg} / \mathrm{m}^{2}\right)$ & 542 & $11 \cdot 4$ & 527 & $11 \cdot 9$ & 759 & $15 \cdot 5$ & 2934 & $59 \cdot 2$ \\
\hline Overweight/obese $\left(\geq 25.0 \mathrm{~kg} / \mathrm{m}^{2}\right)$ & 3059 & $64 \cdot 3$ & 2790 & $63 \cdot 2$ & 2841 & $58 \cdot 1$ & 821 & $16 \cdot 6$ \\
\hline Work status & & & & & & & & \\
\hline At home & 1175 & $24 \cdot 7$ & 1301 & $29 \cdot 5$ & 1812 & $37 \cdot 0$ & 1569 & $31 \cdot 6$ \\
\hline Away from home & 1391 & $29 \cdot 2$ & 1242 & $28 \cdot 1$ & 1413 & $28 \cdot 9$ & 1398 & $28 \cdot 2$ \\
\hline Not working & 2192 & $46 \cdot 1$ & 1870 & $42 \cdot 4$ & 1668 & $34 \cdot 1$ & 1991 & $40 \cdot 2$ \\
\hline Number of children $<5$ years old livi & & & & & & & & \\
\hline 1 & 1296 & $27 \cdot 2$ & 1480 & $33 \cdot 5$ & 1656 & $33 \cdot 8$ & 1597 & $32 \cdot 2$ \\
\hline 2 & 2128 & $44 \cdot 7$ & 2003 & $45 \cdot 4$ & 2149 & $43 \cdot 9$ & 2180 & $44 \cdot 0$ \\
\hline$\geq 3$ & 1333 & $28 \cdot 0$ & 930 & $21 \cdot 1$ & 1088 & $22 \cdot 2$ & 1181 & $23 \cdot 8$ \\
\hline Child-related characteristics & & & & & & & & \\
\hline Sex & & & & & & & & \\
\hline Male & 2371 & $49 \cdot 8$ & 2246 & $50 \cdot 9$ & 2464 & $50 \cdot 4$ & 2523 & $50 \cdot 9$ \\
\hline Female & 2386 & $50 \cdot 2$ & 2167 & $49 \cdot 1$ & 2428 & $49 \cdot 6$ & 2435 & $49 \cdot 1$ \\
\hline Age (months) & & & & & & & & \\
\hline$<6$ & 519 & $10 \cdot 9$ & 522 & $11 \cdot 8$ & 601 & $12 \cdot 3$ & 528 & $10 \cdot 7$ \\
\hline $7-12$ & 559 & $11 \cdot 8$ & 499 & $11 \cdot 3$ & 610 & $12 \cdot 5$ & 566 & $11 \cdot 4$ \\
\hline $13-23$ & 874 & $18 \cdot 4$ & 891 & $20 \cdot 2$ & 922 & $18 \cdot 8$ & 931 & $18 \cdot 8$ \\
\hline 24-35 & 940 & $19 \cdot 8$ & 909 & $20 \cdot 6$ & 913 & $18 \cdot 7$ & 1029 & $20 \cdot 8$ \\
\hline $36-59$ & 1866 & $39 \cdot 2$ & 1592 & $36 \cdot 1$ & 1847 & $37 \cdot 7$ & 1903 & $38 \cdot 4$ \\
\hline Birth order & & & & & & & & \\
\hline 1 & 912 & $19 \cdot 2$ & 1090 & $24 \cdot 6$ & 1124 & $23 \cdot 0$ & 1068 & $21 \cdot 5$ \\
\hline 2 or 3 & 1509 & $31 \cdot 7$ & 1528 & $34 \cdot 5$ & 1775 & $36 \cdot 3$ & 1897 & $38 \cdot 3$ \\
\hline$\geq 4$ & 2337 & $49 \cdot 1$ & 1815 & $40 \cdot 9$ & 1994 & $40 \cdot 8$ & 1993 & $40 \cdot 2$ \\
\hline
\end{tabular}




\begin{tabular}{|c|c|c|c|c|c|c|c|c|}
\hline & \multicolumn{2}{|c|}{$\begin{array}{c}1993 \\
(n 4757)\end{array}$} & \multicolumn{2}{|c|}{$\begin{array}{c}1998 \\
(n \text { 4413) }\end{array}$} & \multicolumn{2}{|c|}{$\begin{array}{c}2003 \\
(n \text { 4893) }\end{array}$} & \multicolumn{2}{|c|}{$\begin{array}{c}2008-2009 \\
(n \text { 4958) }\end{array}$} \\
\hline & $n$ & $\%$ & $n$ & $\%$ & $n$ & $\%$ & $n$ & $\%$ \\
\hline \multicolumn{9}{|l|}{ Size at birth } \\
\hline Small & 732 & $15 \cdot 4$ & 442 & $10 \cdot 0$ & 755 & $15 \cdot 4$ & 781 & $15 \cdot 8$ \\
\hline Average & 2485 & $52 \cdot 2$ & 1744 & $39 \cdot 3$ & 2901 & $59 \cdot 3$ & 2580 & $52 \cdot 0$ \\
\hline Large & 1517 & $31 \cdot 9$ & 645 & $14 \cdot 6$ & 1219 & $24 \cdot 9$ & 1564 & $31 \cdot 5$ \\
\hline \multicolumn{9}{|l|}{ Diarrhoea } \\
\hline Yes & 4072 & $85 \cdot 6$ & 511 & $11 \cdot 5$ & 819 & $16 \cdot 7$ & 866 & $17 \cdot 5$ \\
\hline No & 669 & $14 \cdot 1$ & 2330 & $52 \cdot 6$ & 4065 & $83 \cdot 1$ & 4087 & $82 \cdot 4$ \\
\hline \multicolumn{9}{|l|}{ Fever } \\
\hline Yes & 2708 & $56 \cdot 9$ & 1234 & $27 \cdot 8$ & 2081 & $42 \cdot 5$ & 1222 & $24 \cdot 7$ \\
\hline No & 2031 & $42 \cdot 7$ & 1607 & $36 \cdot 3$ & 2805 & $57 \cdot 3$ & 3727 & $75 \cdot 2$ \\
\hline \multicolumn{9}{|l|}{ Cough } \\
\hline Yes & 2670 & $56 \cdot 1$ & 1108 & $25 \cdot 0$ & 2105 & $43 \cdot 0$ & 1222 & $24 \cdot 7$ \\
\hline No & 2072 & $43 \cdot 5$ & 1733 & $39 \cdot 1$ & 2781 & $56 \cdot 8$ & 3727 & $75 \cdot 2$ \\
\hline
\end{tabular}

†1993 and 1998 surveys excluded North Eastern Province.

¥Excludes 1792 cases with missing data for wealth index in 1993.

$\S$ Excludes 32 cases with missing data for type of water source in 1993.

\|Excludes 14 cases with missing data for type of water source in 1998.

TExcludes 1602 cases with missing data for child morbidity in 1998.

mothers had primary education (Table 3). This pattern of maternal education as a determinant of stunting was repeated in regard to underweight (Table 5). Children of non-educated mothers were $2 \cdot 3$ and $2 \cdot 2$ times more likely to be wasted in 1998 and 2003 compared with those whose mothers had secondary or higher levels of education (Table 4). Children whose mothers were thin $\left(\mathrm{BMI}<18.5 \mathrm{~kg} / \mathrm{m}^{2}\right)$ or pregnant $/$ two months postpartum were significantly more likely to be stunted $(P<0 \cdot 005)$ compared with those whose mothers had a normal BMI (Table 3). Thin mothers were more likely to have wasted and underweight children in all four surveys (Tables 4 and 5). Children whose mothers were overweight/obese $\left(\mathrm{BMI} \geq 25 \cdot 0 \mathrm{~kg} / \mathrm{m}^{2}\right.$ ) were significantly less likely to be stunted in 1998 and $2003(P<0.005$ and $P<0.001$, respectively). This trend shifted in 2008-2009, where children of overweight/obese mothers were significantly more likely $(P<0 \cdot 05)$ to be stunted compared with children of normal-weight mothers (Table 3 ).

\section{Child-related characteristics}

There was a positive association between the child's gender, age and size at birth with undernutrition during the four KDHS (Table 2). Male children were more undernourished in all four surveys. Children aged 24-35 months had significantly higher odds of being stunted or underweight (Table 3 and 5). A significantly higher percentage of stunted, wasted and underweight children were small at birth during the four surveys (all $P<0 \cdot 005$; Table 2). Children who were born small in size were more likely to have undernutrition compared with those born large in all four surveys (Tables 3-5). The level of undernutrition was higher in children who had diarrhoea preceding the surveys compared with those who did not have diarrhoea. This relationship was statistically significant $(P<0.005)$ for stunting in 1993, 1998 and 2003, and statistically significant $(P<0 \cdot 05)$ for wasting and underweight throughout the surveys (Table 2).

\section{Discussion}

The analysis shows a slow decline of chronic undernutrition by 4.6 percentage points as well as a slight decline in underweight (by 2.7 percentage points) in Kenya between 1993 and 2008-2009. The present study is one of the first to analyse the trends of childhood undernutrition in Kenya over three decades using national representative data. The persistent high levels of stunting and underweight are indicators of the slow progress in improving child nutritional status in Kenya. Findings from similar analyses in India, Bangladesh and Mozambique covering more than two decades reported slow national declines in undernutrition among young children $^{(16-18)}$. This slow decline in undernutrition rates in Kenya has continued to undermine efforts geared towards reducing the prevalence of child undernutrition. For instance, the 1999-2004 Kenya Ministry of Health strategic plan ${ }^{(19)}$ targeting to reduce childhood undernutrition by $30 \%$ is far from achievement considering the 1.7 percentage point change in the prevalence of stunted children between 1998 and 2003 as per the current analysis.

The wealth index as used in the DHS compares the population within the same survey year in a relative sense. Thus as applied in the present analysis, it is difficult to compare groups in the same quintiles in different surveys. However it is a useful measure of relative economic differences within the same survey year. Disparities exist in the distribution of undernutrition among Kenyan children based on region (provinces), residence 


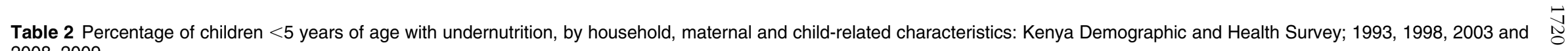
2008-2009

\begin{tabular}{|c|c|c|c|c|c|c|c|c|c|c|c|c|}
\hline & \multicolumn{4}{|c|}{ Stunting (height-for-age $<-2 \mathrm{sD}$ ) } & \multicolumn{4}{|c|}{ Wasting (weight-for-height $<-2 \mathrm{sD}$ ) } & \multicolumn{4}{|c|}{ Underweight (weight-for-age $<-2 \mathrm{sD}$ ) } \\
\hline & 1993 & 1998 & 2003 & 2008-2009 & 1993 & 1998 & 2003 & 2008-2009 & 1993 & 1998 & 2003 & 2008-2009 \\
\hline Total & $39 \cdot 9$ & $37 \cdot 7$ & $36 \cdot 0$ & $35 \cdot 3$ & $6 \cdot 7$ & 6.9 & $6 \cdot 0$ & $6 \cdot 5$ & $18 \cdot 7$ & $18 \cdot 0$ & $15 \cdot 7$ & $16 \cdot 0$ \\
\hline $\begin{array}{l}95 \% \mathrm{Cl} \\
\mathrm{SE}\end{array}$ & $\begin{array}{c}38 \cdot 5,41 \cdot 3 \\
0.007\end{array}$ & $\begin{array}{c}36 \cdot 3,39 \cdot 2 \\
0.007\end{array}$ & $\begin{array}{c}34 \cdot 6,37 \cdot 3 \\
0.007\end{array}$ & $\begin{array}{c}33 \cdot 9,36 \cdot 6 \\
0 \cdot 007\end{array}$ & $\begin{array}{c}6 \cdot 0,7 \cdot 4 \\
0 \cdot 004\end{array}$ & $\begin{array}{c}6 \cdot 1,7 \cdot 6 \\
0.004\end{array}$ & $\begin{array}{c}5 \cdot 3,6 \cdot 6 \\
0.003\end{array}$ & $\begin{array}{c}5 \cdot 9,7 \cdot 2 \\
0.004\end{array}$ & $\begin{array}{c}17 \cdot 6,18 \cdot 7 \\
0 \cdot 006\end{array}$ & $\begin{array}{c}16 \cdot 9,19 \cdot 2 \\
0.006\end{array}$ & $\begin{array}{c}14 \cdot 7,16 \cdot 7 \\
0.005\end{array}$ & $\begin{array}{c}15 \cdot 0,17 \cdot 0 \\
0 \cdot 005\end{array}$ \\
\hline \multicolumn{13}{|c|}{ Household characteristics } \\
\hline \multicolumn{13}{|l|}{ Residence } \\
\hline Rural & $41 \cdot 4$ & $39 \cdot 7$ & $37 \cdot 3$ & $37 \cdot 1$ & $6 \cdot 8$ & $7 \cdot 0$ & $6 \cdot 3$ & $6 \cdot 9$ & $19 \cdot 8$ & $19 \cdot 6$ & $16 \cdot 9$ & $17 \cdot 1$ \\
\hline Urban & $27 \cdot 8$ & $28 \cdot 3$ & $29 \cdot 8$ & $26 \cdot 5$ & $5 \cdot 2$ & $6 \cdot 0$ & $4 \cdot 6$ & $5 \cdot 1$ & $10 \cdot 3$ & $10 \cdot 6$ & $10 \cdot 0$ & $10 \cdot 4$ \\
\hline$P$ value & 0.000 & 0.000 & 0.000 & 0.000 & 0.91 & $0 \cdot 16$ & 0.000 & 0.036 & 0.000 & 0.000 & 0.000 & 0.000 \\
\hline \multicolumn{13}{|l|}{ Province } \\
\hline North Easternt & & & $29 \cdot 2$ & $36 \cdot 1$ & & & $26 \cdot 5$ & $20 \cdot 0$ & & & $30 \cdot 1$ & $25 \cdot 4$ \\
\hline Central & $37 \cdot 6$ & $33 \cdot 4$ & $31 \cdot 1$ & $32 \cdot 8$ & $4 \cdot 8$ & $6 \cdot 1$ & $4 \cdot 5$ & $5 \cdot 2$ & $15 \cdot 0$ & $11 \cdot 7$ & $10 \cdot 8$ & $13 \cdot 3$ \\
\hline Coast & $48 \cdot 1$ & $42 \cdot 9$ & $40 \cdot 9$ & $40 \cdot 2$ & $11 \cdot 9$ & $5 \cdot 5$ & $5 \cdot 5$ & 11 & $29 \cdot 7$ & $22 \cdot 0$ & $19 \cdot 0$ & $24 \cdot 3$ \\
\hline Eastern & $47 \cdot 6$ & $43 \cdot 3$ & $40 \cdot 5$ & $41 \cdot \overline{6}$ & $7 \cdot 8$ & $5 \cdot 3$ & $4 \cdot 3$ & $7 \cdot 0$ & $23 \cdot 6$ & $22 \cdot 7$ & $17 \cdot 2$ & $19 \cdot 9$ \\
\hline Nyanza & 39.5 & $35 \cdot 8$ & $35 \cdot 7$ & $31 \cdot 3$ & $6 \cdot 0$ & $8 \cdot 4$ & $3 \cdot 1$ & 3.9 & $17 \cdot 2$ & $18 \cdot 7$ & $11 \cdot 6$ & $10 \cdot 3$ \\
\hline Rift Valley & $35 \cdot 0$ & $36 \cdot 6$ & $37 \cdot 8$ & $35 \cdot 8$ & $8 \cdot 4$ & $8 \cdot 1$ & $8 \cdot 0$ & $8 \cdot 3$ & $19 \cdot 7$ & $19 \cdot 3$ & $19 \cdot 1$ & $18 \cdot 7$ \\
\hline Western & $36 \cdot 7$ & 39.9 & $35 \cdot 1$ & $32 \cdot 1$ & 3.9 & $4 \cdot 8$ & $6 \cdot 4$ & $2 \cdot 3$ & $12 \cdot 7$ & $15 \cdot 5$ & $16 \cdot 1$ & 10.5 \\
\hline Nairobi & $32 \cdot 1$ & $28 \cdot 5$ & $23 \cdot 7$ & $29 \cdot 1$ & $1 \cdot 8$ & $8 \cdot 4$ & 3.9 & 3.9 & 8.6 & $7 \cdot 6$ & $5 \cdot 2$ & $7 \cdot 8$ \\
\hline$P$ value & 0.000 & 0.000 & 0.000 & 0.000 & 0.000 & 0.017 & 0.000 & 0.000 & 0.000 & 0.000 & 0.000 & 0.000 \\
\hline \multicolumn{13}{|l|}{ Wealth index } \\
\hline Poorest & $43 \cdot 1$ & $49 \cdot 2$ & $44 \cdot 5$ & $44 \cdot 2$ & $10 \cdot 7$ & $7 \cdot 9$ & $9 \cdot 0$ & $11 \cdot 7$ & $22 \cdot 9$ & $26 \cdot 1$ & $24 \cdot 1$ & $24 \cdot 7$ \\
\hline Poorer & $42 \cdot 3$ & $42 \cdot 2$ & $38 \cdot 1$ & $39 \cdot 0$ & $8 \cdot 7$ & $7 \cdot 2$ & $6 \cdot 8$ & $5 \cdot 5$ & $21 \cdot 3$ & $21 \cdot 5$ & $16 \cdot 1$ & $17 \cdot 1$ \\
\hline Middle & $35 \cdot 2$ & $35 \cdot 6$ & $35 \cdot 2$ & $34 \cdot 6$ & $6 \cdot 4$ & 6.5 & $4 \cdot 0$ & $5 \cdot 4$ & $15 \cdot 6$ & $16 \cdot 1$ & $14 \cdot 2$ & $15 \cdot 3$ \\
\hline Richer & 38.0 & $35 \cdot 7$ & $32 \cdot 9$ & $29 \cdot 2$ & $6 \cdot 0$ & $5 \cdot 7$ & $5 \cdot 1$ & 4.9 & $15 \cdot 9$ & $14 \cdot 3$ & $13 \cdot 3$ & $10 \cdot 3$ \\
\hline Richest & $21 \cdot 9$ & $20 \cdot 3$ & $25 \cdot 2$ & $25 \cdot 1$ & $3 \cdot 7$ & $6 \cdot 6$ & $3 \cdot 8$ & 3.5 & $8 \cdot 1$ & $8 \cdot 1$ & $7 \cdot 4$ & $9 \cdot 0$ \\
\hline$P$ value & 0.000 & 0.000 & 0.000 & 0.000 & 0.21 & 0.455 & 0.246 & 0.016 & 0.000 & 0.000 & 0.000 & 0.000 \\
\hline \multicolumn{13}{|c|}{ Source of drinking water } \\
\hline Non-improved & $42 \cdot 4$ & $40 \cdot 4$ & $40 \cdot 3$ & $38 \cdot 8$ & $7 \cdot 8$ & $7 \cdot 1$ & $6 \cdot 2$ & $7 \cdot 7$ & $20 \cdot 1$ & $20 \cdot 0$ & $18 \cdot 0$ & $19 \cdot 2$ \\
\hline Improved & $37 \cdot 3$ & $30 \cdot 4$ & $30 \cdot 7$ & $32 \cdot 5$ & $5 \cdot 6$ & $6 \cdot 2$ & $5 \cdot 5$ & $5 \cdot 6$ & $14 \cdot 3$ & $12 \cdot 6$ & $11 \cdot 0$ & $13 \cdot 4$ \\
\hline$P$ value & 0.000 & 0.000 & 0.000 & 0.000 & 0.001 & 0.172 & 0.177 & 0.002 & 0.000 & 0.000 & 0.000 & 0.000 \\
\hline \multicolumn{13}{|l|}{ Toilet facilities } \\
\hline No toilet facility & $51 \cdot 0$ & $48 \cdot 4$ & $41 \cdot 0$ & $42 \cdot 1$ & $9 \cdot 8$ & 8.9 & $11 \cdot 6$ & $13 \cdot 0$ & $28 \cdot 6$ & $28 \cdot 4$ & $24 \cdot 9$ & $28 \cdot 1$ \\
\hline Non-improved & $40 \cdot 1$ & $38 \cdot 3$ & $37 \cdot 1$ & $39 \cdot 4$ & $6 \cdot 4$ & $6 \cdot 3$ & 4.3 & $5 \cdot 7$ & $17 \cdot 8$ & $17 \cdot 4$ & $14 \cdot 1$ & $16 \cdot 0$ \\
\hline Improved & 21.9 & $21 \cdot 3$ & $23 \cdot 2$ & $27 \cdot 9$ & $4 \cdot 1$ & $1 \cdot 0$ & $4 \cdot 0$ & $4 \cdot 7$ & $10 \cdot 1$ & $7 \cdot 6$ & $8 \cdot 2$ & $10 \cdot 4$ \\
\hline$P$ value & 0.000 & 0.000 & 0.000 & 0.000 & 0.000 & 0.036 & 0.000 & 0.000 & 0.000 & 0.000 & 0.000 & 0.000 \\
\hline \multirow{2}{*}{\multicolumn{13}{|c|}{ Maternal characteristics }} \\
\hline \multicolumn{9}{|c|}{ Mother's age (years) } & & & & \\
\hline$<20$ & $38 \cdot 2$ & $37 \cdot 0$ & $37 \cdot 7$ & $35 \cdot 5$ & $5 \cdot 4$ & $6 \cdot 2$ & $7 \cdot 7$ & $7 \cdot 8$ & $20 \cdot 1$ & $17 \cdot 6$ & $16 \cdot 5$ & $14 \cdot 7$ \\
\hline $20-30$ & 39.6 & $37 \cdot 5$ & $35 \cdot 3$ & 33.9 & $7 \cdot 0$ & $7 \cdot 2$ & $6 \cdot 0$ & $6 \cdot 3$ & $18 \cdot 1$ & $16 \cdot 8$ & $15 \cdot 4$ & $14 \cdot 4$ \\
\hline$>30$ & $40 \cdot 6$ & $38 \cdot 4$ & $36 \cdot 8$ & $37 \cdot 7$ & $6 \cdot 4$ & $6 \cdot 4$ & $5 \cdot 6$ & $6 \cdot 9$ & $19 \cdot 6$ & $20 \cdot 5$ & $16 \cdot 1$ & $19 \cdot 2$ \\
\hline$P$ value & 0.676 & 0.834 & 0.468 & 0.037 & 0.524 & 0.538 & 0.338 & 0.538 & 0.363 & 0.011 & 0.762 & 0.000 \\
\hline \multicolumn{13}{|l|}{ Marital status } \\
\hline Married & $40 \cdot 1$ & $37 \cdot 7$ & $36 \cdot 1$ & $35 \cdot 1$ & $7 \cdot 9$ & $5 \cdot 6$ & $6 \cdot 1$ & 6.5 & $18 \cdot 6$ & $17 \cdot 8$ & $16 \cdot 2$ & $16 \cdot 1$ \\
\hline Single & $38 \cdot 8$ & $38 \cdot 1$ & $35 \cdot 7$ & $35 \cdot 8$ & $6 \cdot 4$ & $7 \cdot 1$ & $5 \cdot 7$ & $6 \cdot 8$ & $19 \cdot 1$ & $19 \cdot 1$ & $15 \cdot 5$ & $15 \cdot 9$ \\
\hline$P$ value & 0.253 & 0.424 & 0.415 & 0.347 & 0.083 & 0.086 & 0.335 & 0.371 & 0.397 & 0.228 & 0.311 & 0.468 \\
\hline \multicolumn{13}{|l|}{ Education } \\
\hline No education & $45 \cdot 0$ & $49 \cdot 6$ & $41 \cdot 2$ & 38.5 & $10 \cdot 7$ & 8.5 & $14 \cdot 5$ & $14 \cdot 0$ & $26 \cdot 0$ & 31.5 & $28 \cdot 7$ & $28 \cdot 0$ \\
\hline Primary & $43 \cdot 3$ & $41 \cdot 3$ & $39 \cdot 3$ & $38 \cdot 2$ & $6 \cdot 4$ & $7 \cdot 6$ & 4.9 & 5.9 & $19 \cdot 4$ & $19 \cdot 4$ & $15 \cdot 6$ & $16 \cdot 7$ \\
\hline Secondary + & $26 \cdot 9$ & $24 \cdot 2$ & $22 \cdot 5$ & $25 \cdot 3$ & $4 \cdot 3$ & $4 \cdot 4$ & 3.5 & $3 \cdot 7$ & $11 \cdot 3$ & $9 \cdot 0$ & $7 \cdot 2$ & $7 \cdot 6$ \\
\hline$P$ value & 0.000 & 0.000 & 0.000 & 0.000 & 0.000 & 0.001 & 0.000 & 0.000 & 0.000 & 0.000 & 0.000 & 0.000 \\
\hline
\end{tabular}


Note: table is based on children of interviewed mothers.

+1993 and 1998 surveys excluded North Eastern Province. 


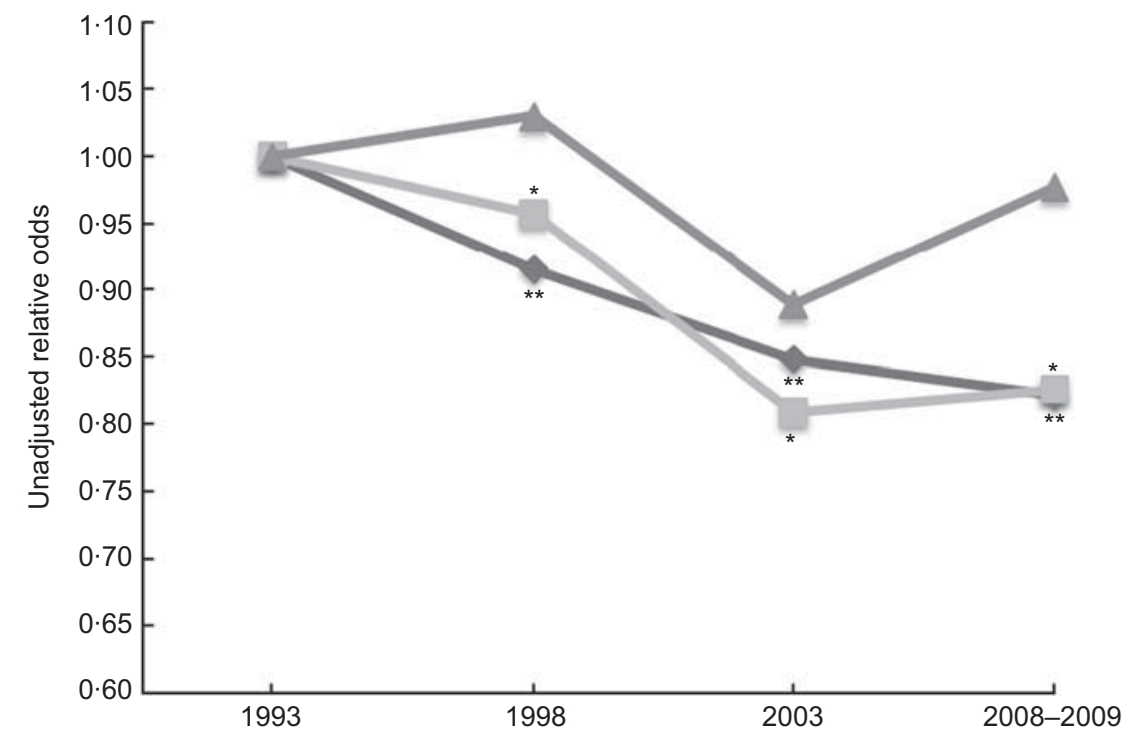

Fig. 1 Trends in unadjusted relative odds of stunting $(=)$, underweight $(=)$ ) and wasting $(=)$ among children $<5$ years of age in Kenya. Model based on pooled data from four Kenya Demographic and Health Surveys (1993, 1998, 2003 and 2008-2009). **Unadjusted odds of stunting were significantly lower in 1998, 2003 and 2008-2009 compared with 1993 (P<0.01); ${ }^{*}$ unadjusted odds of underweight were significantly lower in 2003 and 2008-2009 compared with $1993(P<0.05)$

(rural and urban) and economic divide as measured by the household wealth quintiles. The trend shows a much stronger influence of the wealth index in the earlier surveys in 1993 and 1998 whereby children from the poorest, poorer, middle and richer quintiles were more likely to be stunted compared with the richest. This effect was reduced in 2003 and 2008-2009 when only children from the households with the poorest wealth index were more likely to be stunted compared with the richest. Clearly more efforts need to go into planning effective programmes that target the most vulnerable children. Separately, the availability and type of household toilet facilities was associated with chronic malnutrition. Children living in households with no or non-improved toilet facilities were more likely have short stature when compared with those having improved toilet facilities. Studies in Sub-Saharan Africa have found similar results. In Ethiopia, low child stature was associated with lack of toilet facilities and unprotected water sources ${ }^{(20)}$ while an analysis of DHS data from eleven countries in SubSaharan Africa identified lack of optimal sanitation facilities as a determinant of chronic malnutrition among young children ${ }^{(21)}$. The slight decline noticed may be explained by the increasing social and human development efforts that have been going on in the country in recent years resulting in a marginal increase in the country's Human Development Index ${ }^{(22)}$, an indication of people's improving standards of living. A further analysis of the trends of child nutritional status within the SubSaharan African region could provide further understanding of the regional changes in childhood nutrition indicators as well as explain some of the drivers of the observed slow reduction of undernutrition among Kenyan children.

The levels of acute malnutrition measured by wasting did not change much over the years, remaining at about $6 \%$. This form of malnutrition is caused mainly by recent inadequate food intake, infections or incorrect feeding practices and reflects the nutritional status of children within a short period of time. Kenya has been faced with repeated cycles of famine and drought resulting in insufficient food production at the household level and nationally. This, coupled with the global increases in food prices, has resulted in extended food insecurity in the country that may have contributed to the non-reducing levels of wasted children over the years.

The current study emphasizes the importance of maternal education on child nutrition outcomes in Kenya. These findings agree with results of an analysis by Giroux $^{(23)}$ of the effect of maternal education on child stunting in the Sub-Saharan African region. Attaining a secondary or higher level of maternal education was associated with lower odds of childhood stunting and underweight. Higher maternal education is associated with maternal employment and higher household income. These findings concur with those of Gewa and Yandell $^{(11)}$ who found that low maternal education and poor maternal nutritional status were associated with higher odds of childhood undernutrition in Kenya. The effect of maternal education on childhood stunting and underweight is, however, changing over time in Kenya. In the earlier surveys (1993, 1998 and 2003), lack of education among mothers as well as attaining primary level of education were associated with poor child nutritional 
Table 3 Relationship between childhood stunting and household, maternal and child-related characteristics: Kenya Demographic and Health Survey; 1993, 1998, 2003 and 2008-2009

\begin{tabular}{|c|c|c|c|c|c|c|c|c|}
\hline \multirow[b]{2}{*}{ Factor } & \multicolumn{2}{|c|}{1993} & \multicolumn{2}{|c|}{1998} & \multicolumn{2}{|c|}{2003} & \multicolumn{2}{|c|}{$2008-2009$} \\
\hline & OR & $95 \% \mathrm{Cl}$ & OR & $95 \% \mathrm{Cl}$ & OR & $95 \% \mathrm{Cl}$ & OR & $95 \% \mathrm{Cl}$ \\
\hline \multirow{2}{*}{\multicolumn{9}{|c|}{$\begin{array}{l}\text { Household characteristics } \\
\text { Residence }\end{array}$}} \\
\hline & & \multicolumn{5}{|c|}{ Residence } & & \\
\hline Rural & 0.9 & $0 \cdot 6,1 \cdot 3$ & $1 \cdot 1$ & $0 \cdot 7,1 \cdot 7$ & $0.7^{*}$ & $0 \cdot 6,1 \cdot 0$ & $1 \cdot 1$ & $0 \cdot 8,1 \cdot 6$ \\
\hline Urban (ref.) & $1 \cdot 0$ & & $1 \cdot 0$ & & $1 \cdot 0$ & & $1 \cdot 0$ & \\
\hline \multicolumn{9}{|l|}{ Province } \\
\hline North Easternt & & & & & $0.5^{*}$ & $0.3,0.9$ & $0.5^{\star \star}$ & $0.3,0.8$ \\
\hline Central & 0.6 & $0 \cdot 3,1 \cdot 1$ & $0 \cdot 7$ & $0 \cdot 4,1 \cdot 5$ & $1 \cdot 1$ & $0 \cdot 7,1 \cdot 7$ & $0 \cdot 7$ & $0 \cdot 4,1 \cdot 2$ \\
\hline Coast & 0.5 & $0 \cdot 3,1 \cdot 0$ & $0 \cdot 8$ & $0 \cdot 4,1 \cdot 6$ & $1 \cdot 3$ & $0.8,1.9$ & 0.8 & $0.4,1.5$ \\
\hline Eastern & 0.6 & $0 \cdot 3,1 \cdot 1$ & $0 \cdot 8$ & $0 \cdot 4,1 \cdot 4$ & $1 \cdot 3$ & $0 \cdot 8,2 \cdot 0$ & $0 \cdot 7$ & $0 \cdot 4,1 \cdot 2$ \\
\hline Nyanza & $0.5^{\star}$ & $0.3,0.9$ & $0 \cdot 6$ & $0 \cdot 3,1 \cdot 1$ & $1 \cdot 0$ & $0 \cdot 6,1 \cdot 6$ & $0.5^{\star *}$ & $0 \cdot 3,0 \cdot 8$ \\
\hline Rift Valley & $0 \cdot 4^{\star \star}$ & $0 \cdot 2,0 \cdot 7$ & $0 \cdot 6$ & $0 \cdot 3,1$ & $1 \cdot 1$ & $0 \cdot 7,1 \cdot 7$ & $0 \cdot 5^{\star}$ & $0 \cdot 3,0 \cdot 9$ \\
\hline Western & $0 \cdot 4^{\star \star}$ & $0 \cdot 2,0.7$ & $0 \cdot 7$ & $0 \cdot 4,1 \cdot 4$ & 0.9 & $0 \cdot 6,1 \cdot 4$ & $0 \cdot 4^{\star *}$ & $0 \cdot 2,0 \cdot 7$ \\
\hline Nairobi (ref.) & $1 \cdot 0$ & & $1 \cdot 0$ & & $1 \cdot 0$ & & $1 \cdot 0$ & \\
\hline Wealth index & & & & & & & & \\
\hline Poorest & $2 \cdot 1^{\star \star}$ & $1 \cdot 3,3 \cdot 3$ & $2 \cdot 6^{\star \star \star}$ & $1 \cdot 6,4 \cdot 3$ & $1 \cdot 6^{*}$ & $1 \cdot 1,2 \cdot 4$ & $2 \cdot 1^{\star \star}$ & $1 \cdot 4,3 \cdot 1$ \\
\hline Poorer & $2 \cdot 1^{\star \star}$ & $1 \cdot 4,3 \cdot 3$ & $2 \cdot 0^{\star \star}$ & $1 \cdot 3,3 \cdot 2$ & $1 \cdot 3$ & $0.9,1.9$ & 1.5 & $1 \cdot 0,2 \cdot 1$ \\
\hline Middle & $1 \cdot 7^{\star}$ & $1 \cdot 1,2 \cdot 6$ & $1 \cdot 7^{\star}$ & $1 \cdot 1,2 \cdot 6$ & $1 \cdot 2$ & $0 \cdot 8,1 \cdot 7$ & $1 \cdot 3$ & $0 \cdot 9,2 \cdot 0$ \\
\hline Richer & $1 \cdot 6^{*}$ & $1 \cdot 1,2 \cdot 3$ & $1 \cdot 7^{*}$ & $1 \cdot 1,2 \cdot 7$ & $1 \cdot 1$ & $0.8,1 \cdot 6$ & $1 \cdot 2$ & $0.9,1 \cdot 8$ \\
\hline Richest (ref.) & $1 \cdot 0$ & & $1 \cdot 0$ & & $1 \cdot 0$ & & $1 \cdot 0$ & \\
\hline Source of drinking water & & & & & & & & \\
\hline Non-improved & 0.8 & $0 \cdot 6,1 \cdot 1$ & 0.9 & $0 \cdot 7,1 \cdot 2$ & $1 \cdot 2$ & $1 \cdot 0,1 \cdot 8$ & $1 \cdot 1$ & $0 \cdot 8,1 \cdot 2$ \\
\hline Improved (ref.) & $1 \cdot 0$ & & $1 \cdot 0$ & & $1 \cdot 0$ & & $1 \cdot 0$ & \\
\hline Toilet facilities & & & & & & & & \\
\hline No toilet facility & $2 \cdot 2^{\star *}$ & $1 \cdot 3,3 \cdot 7$ & $1 \cdot 4$ & $0 \cdot 9,2 \cdot 1$ & $1 \cdot 1$ & $0.8,1 \cdot 6$ & $1 \cdot 1$ & $0.8,1.5$ \\
\hline Non-improved & $1 \cdot 5^{\star}$ & $1 \cdot 0,2 \cdot 3$ & $1 \cdot 3$ & $0 \cdot 9,1 \cdot 8$ & $1 \cdot 4^{*}$ & $1 \cdot 0,1 \cdot 8$ & $1 \cdot 3^{\star \star}$ & $1 \cdot 1,1 \cdot 6$ \\
\hline Improved (ref.) & $1 \cdot 0$ & & $1 \cdot 0$ & & $1 \cdot 0$ & & $1 \cdot 0$ & \\
\hline Maternal characteristics & & & & & & & & \\
\hline Mother's age (years) & & & & & & & & \\
\hline$<20$ & $1 \cdot 2$ & $0 \cdot 7,2 \cdot 1$ & $1 \cdot 5$ & $0 \cdot 9,2 \cdot 5$ & $1 \cdot 8^{\star *}$ & $1 \cdot 2,2 \cdot 8$ & $1 \cdot 1$ & $0 \cdot 7,1 \cdot 8$ \\
\hline $20-30$ years & $1 \cdot 0$ & $0 \cdot 8,1 \cdot 3$ & $1 \cdot 2$ & $0.9,1.5$ & $1 \cdot 0$ & $0 \cdot 8,1 \cdot 3$ & 0.9 & $0 \cdot 7,1 \cdot 2$ \\
\hline$>30$ (ref.) & $1 \cdot 0$ & & 1.0 & & $1 \cdot 0$ & & $1 \cdot 0$ & \\
\hline Education & & & & & & & & \\
\hline No education & $1 \cdot 6^{*}$ & $1 \cdot 1,2 \cdot 3$ & $1 \cdot 7^{\star \star}$ & $1 \cdot 2,2 \cdot 5$ & $1 \cdot 7^{\star \star}$ & $1 \cdot 2,2 \cdot 4$ & 0.9 & $0 \cdot 7,1 \cdot 3$ \\
\hline Primary & $1 \cdot 7^{\star \star \star}$ & $1 \cdot 3,2 \cdot 2$ & $1 \cdot 5^{\star \star}$ & $1 \cdot 2,1 \cdot 9$ & $1 \cdot 7^{\star \star \star}$ & $1 \cdot 4,2 \cdot 2$ & $1 \cdot 3^{*}$ & $1 \cdot 1,1 \cdot 7$ \\
\hline Secondary + (ref.) & $1 \cdot 0$ & & $1 \cdot 0$ & & $1 \cdot 0$ & & $1 \cdot 0$ & \\
\hline BMI category & & & & & & & & \\
\hline Pregnant and postpartum & $1 \cdot 5^{\star \star}$ & $1 \cdot 1,1 \cdot 9$ & $1 \cdot 3$ & $1 \cdot 0,1 \cdot 7$ & $1 \cdot 2^{*}$ & $1 \cdot 0,1 \cdot 5$ & $1 \cdot 5^{\star}$ & $1 \cdot 0,2 \cdot 1$ \\
\hline Thin $\left(<18.5 \mathrm{~kg} / \mathrm{m}^{2}\right)$ & $1 \cdot 4^{*}$ & $1 \cdot 0,2 \cdot 0$ & $1 \cdot 2$ & $0.9,1.6$ & $1 \cdot 2^{*}$ & $1 \cdot 0,1 \cdot 5$ & $1 \cdot 7^{\star \star}$ & $1 \cdot 2,2 \cdot 5$ \\
\hline Overweight/obese $\left(\geq 25 \cdot 0 \mathrm{~kg} / \mathrm{m}^{2}\right)$ & 0.8 & $0.6,1 \cdot 2$ & $0.5^{\star \star}$ & $0.4,0.8$ & $0 \cdot 6^{\star \star \star}$ & $0.5,0.8$ & $1 \cdot 3^{\star}$ & $1,1 \cdot 7$ \\
\hline Normal $\left(18 \cdot 5-24.9 \mathrm{~kg} / \mathrm{m}^{2}\right)$ (ref.) & $1 \cdot 0$ & & $1 \cdot 0$ & & $1 \cdot 0$ & & 1.0 & \\
\hline Number of children $<5$ years old livir & & & & & & & & \\
\hline 1 & $0.7^{*}$ & $0.5,1 \cdot 0$ & 0.9 & $0 \cdot 7,1 \cdot 3$ & $0.7^{*}$ & $0 \cdot 6,0 \cdot 9$ & $0.8^{*}$ & $0 \cdot 6,1 \cdot 0$ \\
\hline 2 & $1 \cdot 0$ & $0.8,1 \cdot 3$ & $1 \cdot 0$ & $0 \cdot 7,1 \cdot 3$ & $1 \cdot 0$ & $0 \cdot 8,1 \cdot 2$ & $1 \cdot 0$ & $0.8,1.3$ \\
\hline$\geq 3$ (ref.) & $1 \cdot 0$ & & $1 \cdot 0$ & & $1 \cdot 0$ & & $1 \cdot 0$ & \\
\hline Child-related characteristics & & & & & & & & \\
\hline Sex & & & & & & & & \\
\hline Male & $1 \cdot 5^{\star \star \star}$ & $1 \cdot 3,1 \cdot 8$ & $1 \cdot 6^{\star \star \star}$ & $1 \cdot 3,1 \cdot 9$ & $1 \cdot 5^{\star \star \star}$ & $1 \cdot 3,1 \cdot 7$ & $1 \cdot 3^{\star \star}$ & $1 \cdot 1,1 \cdot 5$ \\
\hline Female (ref.) & $1 \cdot 0$ & & $1 \cdot 0$ & & $1 \cdot 0$ & & $1 \cdot 0$ & \\
\hline Age (months) & & & & & & & & \\
\hline$<6$ months & $0 \cdot 1^{\star \star \star}$ & $0.1,0.2$ & $0 \cdot 1^{\star \star *}$ & $0 \cdot 1,0 \cdot 2$ & $0 \cdot 1^{\star \star *}$ & $0 \cdot 1,0 \cdot 2$ & $0 \cdot 1^{\star \star *}$ & $0 \cdot 1,0 \cdot 2$ \\
\hline $7-12$ & $0 \cdot 3^{\star \star \star}$ & $0.2,0.4$ & $0.3^{\star \star \star}$ & $0.2,0.4$ & $0 \cdot 2^{\star \star \star}$ & $0.2,0.3$ & $0.5^{\star \star \star}$ & $0.3,0.6$ \\
\hline $13-23$ & $0 \cdot 7^{\star \star}$ & $0.5,0.9$ & $0.7^{* \star *}$ & $0.5,0.8$ & 0.9 & $0 \cdot 7,1 \cdot 1$ & $1 \cdot 0$ & $0.8,1 \cdot 4$ \\
\hline $24-35$ (ref.) & $1 \cdot 0$ & & $1 \cdot 0$ & & $1 \cdot 0$ & & $1 \cdot 0$ & \\
\hline $36-59$ & $0 \cdot 6^{* *}$ & $0.5,0.8$ & $0.7^{\star \star \star}$ & $0 \cdot 6,0.8$ & $0 \cdot 6^{\star \star \star}$ & $0.5,0.8$ & $0 \cdot 6^{\star \star \star}$ & $0.5,0.7$ \\
\hline Birth order & & & & & & & & \\
\hline 1 & 0.8 & $0.5,1 \cdot 2$ & $0 \cdot 7$ & $0 \cdot 5,1 \cdot 0$ & $0 \cdot 7^{\star *}$ & $0.5,0.9$ & $2 \cdot 0^{\star \star *}$ & $1 \cdot 5,2 \cdot 6$ \\
\hline 2 or 3 & 0.9 & $0 \cdot 7,1 \cdot 2$ & 0.9 & $0 \cdot 7,1 \cdot 1$ & 0.9 & $0 \cdot 7,1 \cdot 1$ & $1 \cdot 3^{* \star}$ & $1 \cdot 1,1 \cdot 6$ \\
\hline$\geq 4$ (ref.) & $1 \cdot 0$ & & $1 \cdot 0$ & & $1 \cdot 0$ & & $1 \cdot 0$ & \\
\hline Size at birth & & & & & & & & \\
\hline Small & $2 \cdot 2^{\star \star \star}$ & $1 \cdot 6,3 \cdot 0$ & $2 \cdot 0^{\star \star \star}$ & $1 \cdot 5,2 \cdot 9$ & $2 \cdot 1^{\star \star \star}$ & $1 \cdot 6,2 \cdot 6$ & 0.9 & $0 \cdot 7,1 \cdot 2$ \\
\hline Average & $1 \cdot 4^{\star \star}$ & $1 \cdot 1,1 \cdot 7$ & $1 \cdot 2$ & $1 \cdot 0,1 \cdot 5$ & $1 \cdot 4^{\star \star \star}$ & $1 \cdot 2,1 \cdot 7$ & $0 \cdot 8$ & $0 \cdot 6,1 \cdot 1$ \\
\hline Large (ref.) & $1 \cdot 0$ & & $1 \cdot 0$ & & $1 \cdot 0$ & & $1 \cdot 0$ & \\
\hline Diarrhoea & & & & & & & & \\
\hline Had diarrhoea & $0.7^{\star}$ & $0.5,0.9$ & $1 \cdot 6^{\star \star}$ & $1 \cdot 2,2 \cdot 0$ & $1 \cdot 1$ & $0 \cdot 9,1 \cdot 3$ & $1 \cdot 0$ & $0 \cdot 8,1 \cdot 2$ \\
\hline No diarrhoea (ref.) & $1 \cdot 0$ & & $1 \cdot 0$ & & $1 \cdot 0$ & & $1 \cdot 0$ & \\
\hline Fever & & & & & & & & \\
\hline Had fever & $1 \cdot 1$ & $0.9,1 \cdot 4$ & $1 \cdot 1$ & $0 \cdot 9,1 \cdot 4$ & 0.9 & $0 \cdot 8,1 \cdot 1$ & $1 \cdot 0$ & $0 \cdot 8,1 \cdot 2$ \\
\hline Fever (ref.) & $1 \cdot 0$ & & $1 \cdot 0$ & & $1 \cdot 0$ & & $1 \cdot 0$ & \\
\hline Cough & & & & & & & & \\
\hline Had cough & 0.9 & $0 \cdot 8,1 \cdot 1$ & $1 \cdot 0$ & $0 \cdot 8,1 \cdot 3$ & $1 \cdot 2^{*}$ & $1 \cdot 0,1 \cdot 4$ & $1 \cdot 0$ & $0 \cdot 8,1 \cdot 2$ \\
\hline No cough (ref.) & $1 \cdot 0$ & & $1 \cdot 0$ & & $1 \cdot 0$ & & $1 \cdot 0$ & \\
\hline
\end{tabular}

ref., reference category.

Note: table is based on results of adjusted binary logistic regression model.

${ }^{\star} P<0.05,{ }^{* \star} P<0.005,{ }^{* \star *} P<0.001$.

†1993 and 1998 surveys excluded North Eastern Province. 
Table 4 Relationship between childhood wasting and household, maternal and child-related characteristics: Kenya Demographic and Health Survey; 1993, 1998, 2003 and 2008-2009

\begin{tabular}{|c|c|c|c|c|c|c|c|c|}
\hline \multirow[b]{2}{*}{ Factor } & \multicolumn{2}{|c|}{1993} & \multicolumn{2}{|c|}{1998} & \multicolumn{2}{|c|}{2003} & \multicolumn{2}{|c|}{ 2008-2009 } \\
\hline & OR & $95 \% \mathrm{Cl}$ & OR & $95 \% \mathrm{Cl}$ & OR & $95 \% \mathrm{Cl}$ & OR & $95 \% \mathrm{Cl}$ \\
\hline \multicolumn{9}{|l|}{ Household characteristics } \\
\hline \multicolumn{9}{|l|}{ Residence } \\
\hline Rural & 0.7 & $0 \cdot 4,1 \cdot 5$ & $2 \cdot 2^{\star \star}$ & $1 \cdot 2,3 \cdot 9$ & $1 \cdot 1$ & $0 \cdot 6,2 \cdot 0$ & $0 \cdot 6^{*}$ & $0 \cdot 3,1 \cdot 0$ \\
\hline Urban (ref.) & $1 \cdot 0$ & & $1 \cdot 0$ & & $1 \cdot 0$ & & $1 \cdot 0$ & \\
\hline \multicolumn{9}{|l|}{ Province } \\
\hline North Easternt & & & & & $2 \cdot 2$ & $0 \cdot 7,6 \cdot 7$ & $2 \cdot 4$ & $0 \cdot 8,7 \cdot 4$ \\
\hline Central & $1 \cdot 3$ & $0 \cdot 3,5 \cdot 5$ & 0.4 & $0 \cdot 1,1 \cdot 1$ & $1 \cdot 1$ & $0 \cdot 4,2 \cdot 9$ & $1 \cdot 2$ & $0 \cdot 4,3 \cdot 8$ \\
\hline Coast & $3 \cdot 7$ & $0.9,14.9$ & $0.4^{*}$ & $0 \cdot 1,1 \cdot 0$ & 0.5 & $0 \cdot 2,1 \cdot 3$ & $1 \cdot 7$ & $0.6,4 \cdot 8$ \\
\hline Eastern & 1.9 & $0 \cdot 4,8 \cdot 0$ & $0 \cdot 4^{*}$ & $0 \cdot 1,0.9$ & $0 \cdot 7$ & $0 \cdot 2,1 \cdot 9$ & $1 \cdot 3$ & $0.4,3.9$ \\
\hline Nyanza & $1 \cdot 5$ & $0 \cdot 3,6 \cdot 2$ & 0.8 & $0 \cdot 3,1 \cdot 9$ & 0.4 & $0 \cdot 1,1 \cdot 1$ & $0 \cdot 8$ & $0 \cdot 2,2 \cdot 3$ \\
\hline Rift Valley & $1 \cdot 8$ & $0 \cdot 4,7 \cdot 7$ & $0 \cdot 4^{*}$ & $0 \cdot 2,1 \cdot 0$ & 0.9 & $0 \cdot 4,2 \cdot 3$ & $1 \cdot 3$ & $0.5,3 \cdot 8$ \\
\hline Western & $0 \cdot 8$ & $0 \cdot 2,3 \cdot 7$ & $0 \cdot 3^{*}$ & $0 \cdot 1,0 \cdot 9$ & $1 \cdot 2$ & $0.5,2 \cdot 9$ & 0.3 & $0 \cdot 1,1 \cdot 2$ \\
\hline Nairobi (ref.) & $1 \cdot 0$ & & $1 \cdot 0$ & & $1 \cdot 0$ & & $1 \cdot 0$ & \\
\hline \multicolumn{9}{|l|}{ Wealth index } \\
\hline Poorest & 1.5 & $0 \cdot 7,3 \cdot 3$ & $0 \cdot 8$ & $0.3,1.9$ & $1 \cdot 1$ & $0 \cdot 5,2 \cdot 6$ & $3 \cdot 8^{* *}$ & $1 \cdot 7,8 \cdot 5$ \\
\hline Poorer & $1 \cdot 3$ & $0 \cdot 6,2 \cdot 7$ & 0.9 & $0.4,1.9$ & $1 \cdot 3$ & $0 \cdot 6,3 \cdot 0$ & $2 \cdot 4^{\star}$ & $1 \cdot 1,5 \cdot 2$ \\
\hline Middle & $1 \cdot 1$ & $0 \cdot 5,2 \cdot 4$ & 0.6 & $0 \cdot 3,1 \cdot 4$ & $0 \cdot 8$ & $0 \cdot 4,1 \cdot 9$ & $2 \cdot 2^{*}$ & $1 \cdot 1,4 \cdot 7$ \\
\hline Richer & $1 \cdot 0$ & $0 \cdot 5,2 \cdot 1$ & 0.6 & $0 \cdot 3,1 \cdot 3$ & $1 \cdot 2$ & $0 \cdot 6,2 \cdot 4$ & $1 \cdot 7$ & $0.9,3.2$ \\
\hline Richest (ref.) & $1 \cdot 0$ & & $1 \cdot 0$ & & $1 \cdot 0$ & & $1 \cdot 0$ & \\
\hline \multicolumn{9}{|l|}{ Source of drinking water } \\
\hline Non-improved & $1 \cdot 3$ & $0 \cdot 8,2 \cdot 2$ & 0.9 & $0.5,1.5$ & $1 \cdot 1$ & $0 \cdot 8,1 \cdot 6$ & $1 \cdot 0$ & $0 \cdot 7,1 \cdot 4$ \\
\hline Improved (ref.) & $1 \cdot 0$ & & $1 \cdot 0$ & & $1 \cdot 0$ & & $1 \cdot 0$ & $1 \cdot 0$ \\
\hline \multicolumn{9}{|l|}{ Toilet facilities } \\
\hline No toilet facility & $1 \cdot 6$ & $0 \cdot 6,3 \cdot 8$ & $1 \cdot 0$ & $0 \cdot 4,2 \cdot 1$ & 1.5 & $0 \cdot 7,3 \cdot 0$ & $1 \cdot 0$ & $0 \cdot 6,1 \cdot 7$ \\
\hline Non-improved & 1.6 & $0.7,3.5$ & $1 \cdot 0$ & $0.5,1.9$ & 0.8 & $0.5,1.5$ & $1 \cdot 0$ & $0.6,1.5$ \\
\hline Improved (ref.) & $1 \cdot 0$ & & $1 \cdot 0$ & & $1 \cdot 0$ & & $1 \cdot 0$ & $1 \cdot 0$ \\
\hline \multicolumn{9}{|l|}{ Maternal characteristics } \\
\hline \multicolumn{9}{|l|}{ Education } \\
\hline No education & $1 \cdot 7$ & $0.9,3 \cdot 2$ & $2 \cdot 3^{*}$ & $1 \cdot 2,4 \cdot 5$ & $2 \cdot 2^{*}$ & $1 \cdot 1,4 \cdot 4$ & $1 \cdot 6$ & $0 \cdot 9,3 \cdot 1$ \\
\hline Primary & $1 \cdot 3$ & $0 \cdot 8,2 \cdot 2$ & $2 \cdot 1^{\star \star}$ & $1 \cdot 4,3 \cdot 3$ & $1 \cdot 1$ & $0 \cdot 7,1 \cdot 9$ & $1 \cdot 3$ & $0 \cdot 8,2 \cdot 0$ \\
\hline Secondary + (ref.) & $1 \cdot 0$ & & $1 \cdot 0$ & & $1 \cdot 0$ & & $1 \cdot 0$ & \\
\hline \multicolumn{9}{|l|}{ BMI category } \\
\hline Pregnant and postpartum & 0.7 & $0 \cdot 4,1 \cdot 2$ & 0.8 & $0.5,1 \cdot 4$ & 1.5 & $1 \cdot 2, \cdot 2 \cdot 0$ & $0 \cdot 8$ & $0.5,1 \cdot 3$ \\
\hline Thin $\left(<18.5 \mathrm{~kg} / \mathrm{m}^{2}\right)$ & $1 \cdot 9^{\star *}$ & $1 \cdot 2,2 \cdot 9$ & $1 \cdot 7^{*}$ & $1 \cdot 1,2 \cdot 5$ & $2 \cdot 5^{\star \star \star}$ & $1 \cdot 7,3 \cdot 9$ & $1 \cdot 4$ & $1,2 \cdot 1$ \\
\hline Overweight/obese $\left(\geq 25.0 \mathrm{~kg} / \mathrm{m}^{2}\right)$ & 0.7 & $0 \cdot 3,1 \cdot 3$ & $1 \cdot 0$ & $0.5,1 \cdot 8$ & 0.9 & $0.5,1.6$ & 0.9 & $0.5,1.5$ \\
\hline Normal $\left(18.5-24.9 \mathrm{~kg} / \mathrm{m}^{2}\right)$ (ref.) & $1 \cdot 0$ & & $1 \cdot 0$ & & $1 \cdot 0$ & & $1 \cdot 0$ & \\
\hline \multicolumn{9}{|l|}{ Child-related characteristics } \\
\hline Sex & & & & & & & & \\
\hline Male & $1 \cdot 2$ & $0.9,1.7$ & $1 \cdot 1$ & $0 \cdot 8,1 \cdot 5$ & $1 \cdot 6^{\star \star}$ & $1 \cdot 2,2 \cdot 2$ & $1 \cdot 4$ & $1 \cdot 0,1 \cdot 9$ \\
\hline Female (ref.) & $1 \cdot 0$ & & $1 \cdot 0$ & & $1 \cdot 0$ & & $1 \cdot 0$ & \\
\hline Age (months) & & & & & & & & \\
\hline$<6$ & $1 \cdot 8^{*}$ & $1 \cdot 0,3 \cdot 3$ & $3 \cdot 3^{\star \star \star}$ & $2 \cdot 0,5 \cdot 4$ & $1 \cdot 7$ & $1 \cdot 0,2 \cdot 8$ & $1 \cdot 6$ & $1 \cdot 0,2 \cdot 8$ \\
\hline $7-12$ & $1 \cdot 7$ & $0 \cdot 9,3 \cdot 0$ & $2 \cdot 7^{\star \star \star}$ & $1 \cdot 6,4 \cdot 5$ & $2 \cdot 0^{\star \star}$ & $1 \cdot 2,3 \cdot 3$ & $1 \cdot 4$ & $0.8,2 \cdot 5$ \\
\hline $13-23$ & $1 \cdot 4$ & $0.8,2.5$ & $1 \cdot 9^{\star \star}$ & $1 \cdot 2,2 \cdot 9$ & $1 \cdot 7^{\star}$ & $1 \cdot 1,2 \cdot 7$ & 0.8 & $0.5,1.3$ \\
\hline 24-35 (ref.) & $1 \cdot 0$ & & $1 \cdot 0$ & & $1 \cdot 0$ & & $1 \cdot 0$ & \\
\hline $36-59$ & $1 \cdot 2$ & $0 \cdot 6,2 \cdot 2$ & $1 \cdot 3$ & $0 \cdot 8,2 \cdot 1$ & $1 \cdot 0$ & $0.7,1.5$ & 0.8 & $0 \cdot 5,1 \cdot 2$ \\
\hline Birth order & & & & & & & & \\
\hline 1 & 0.5 & $0 \cdot 2,1 \cdot 1$ & $1 \cdot 0$ & $0 \cdot 5,1 \cdot 8$ & $1 \cdot 1$ & $0.6,1.9$ & $1 \cdot 2$ & $0.7,1.9$ \\
\hline 2 or 3 & 0.7 & $0 \cdot 4,1 \cdot 0$ & $1 \cdot 1$ & $0 \cdot 7,1 \cdot 7$ & $1 \cdot 0$ & $0.6,1.6$ & $1 \cdot 0$ & $0 \cdot 6,1 \cdot 5$ \\
\hline$\geq 4$ (ref.) & $1 \cdot 0$ & & $1 \cdot 0$ & & $1 \cdot 0$ & & $1 \cdot 0$ & \\
\hline Size at birth & & & & & & & & \\
\hline Small & $1 \cdot 6^{*}$ & $1 \cdot 0,2 \cdot 6$ & $1 \cdot 3$ & $0 \cdot 8,2 \cdot 1$ & 1.5 & $1 \cdot 0,2 \cdot 3$ & $1 \cdot 8^{* *}$ & $1 \cdot 2,2 \cdot 7$ \\
\hline Average & $1 \cdot 0$ & $0.7,1.5$ & $1 \cdot 2$ & $0 \cdot 8,1 \cdot 8$ & $1 \cdot 2$ & $0 \cdot 8,1 \cdot 7$ & $1 \cdot 0$ & $0 \cdot 7,1 \cdot 3$ \\
\hline Large (ref.) & $1 \cdot 0$ & & $1 \cdot 0$ & & $1 \cdot 0$ & & $1 \cdot 0$ & \\
\hline Diarrhoea & & & & & & & & \\
\hline Yes & $0 \cdot 5^{\star *}$ & $0 \cdot 4,0.8$ & $1 \cdot 5^{\star}$ & $1 \cdot 2, \cdot 2 \cdot 0$ & $1 \cdot 8^{\star *}$ & $1 \cdot 3,2 \cdot 6$ & $1 \cdot 3$ & $1 \cdot 0,1 \cdot 8$ \\
\hline No (ref.) & $1 \cdot 0$ & & $1 \cdot 0$ & & $1 \cdot 0$ & & $1 \cdot 0$ & \\
\hline
\end{tabular}

ref., reference category.

Note: table is based on results of adjusted binary logistic regression model. ${ }^{\star} P<0.05,{ }^{\star \star} P<0.005,{ }^{\star \star \star} P<0.001$.

†1993 and 1998 surveys excluded North Eastern Province.

outcomes, while in the recent survey this effect was noticed among children of mothers with primary level education. This is in line with the changing dynamics of education in the country with the introduction of free primary education in 2002. There has been a reduction in the proportion of mothers without education in the 
Table 5 Relationship between childhood underweight and household, maternal and child-related characteristics: Kenya Demographic and Health Survey; 1993, 1998, 2003 and 2008-2009

\begin{tabular}{|c|c|c|c|c|c|c|c|c|}
\hline \multirow[b]{2}{*}{ Factor } & \multicolumn{2}{|c|}{1993} & \multicolumn{2}{|c|}{1998} & \multicolumn{2}{|c|}{2003} & \multicolumn{2}{|c|}{ 2008-2009 } \\
\hline & OR & $95 \% \mathrm{Cl}$ & OR & $95 \% \mathrm{Cl}$ & OR & $95 \% \mathrm{Cl}$ & OR & $95 \% \mathrm{Cl}$ \\
\hline \multicolumn{9}{|l|}{$\begin{array}{l}\text { Household characteristics } \\
\text { Residence }\end{array}$} \\
\hline $\begin{array}{l}\text { Residence } \\
\text { Rural }\end{array}$ & $1 \cdot 4$ & \multirow{2}{*}{$0 \cdot 7,2 \cdot 5$} & $1 \cdot 3$ & \multirow{2}{*}{$0 \cdot 8,2 \cdot 2$} & 0.9 & \multirow{2}{*}{$0 \cdot 6,1 \cdot 3$} & 0.9 & \multirow{2}{*}{$0 \cdot 6,1 \cdot 3$} \\
\hline Urban (ref.) & 1.0 & & 1.0 & & 1.0 & & 1.0 & \\
\hline \multicolumn{9}{|l|}{ Province } \\
\hline North Easternt & & & & & 2 & $0.8,4.9$ & $1 \cdot 2$ & $0 \cdot 6,2 \cdot 3$ \\
\hline Central & $0 \cdot 7$ & $0 \cdot 3,1 \cdot 8$ & $0 \cdot 6$ & $0 \cdot 2,2 \cdot 0$ & $1 \cdot 6$ & $0 \cdot 7,3 \cdot 8$ & $1 \cdot 3$ & $0 \cdot 6,2 \cdot 6$ \\
\hline Coast & $1 \cdot 2$ & $0.5,2 \cdot 8$ & $1 \cdot 0$ & $0.3,2.9$ & $1 \cdot 6$ & $0 \cdot 7,3 \cdot 6$ & $1 \cdot 7$ & $0 \cdot 8,3 \cdot 7$ \\
\hline Eastern & 0.8 & $0 \cdot 3,2 \cdot 0$ & $1 \cdot 1$ & $0 \cdot 4,3 \cdot 4$ & $2 \cdot 0$ & $0.9,4.5$ & $1 \cdot 3$ & $0 \cdot 7,2 \cdot 7$ \\
\hline Nyanza & $0 \cdot 7$ & $0 \cdot 3,1 \cdot 7$ & $1 \cdot 6$ & $0.5,4.7$ & $1 \cdot 1$ & $0.5,2 \cdot 6$ & 0.8 & $0 \cdot 4,1 \cdot 6$ \\
\hline Rift Valley & $0 \cdot 7$ & $0 \cdot 3,1 \cdot 6$ & $0 \cdot 8$ & $0 \cdot 3,2 \cdot 4$ & $1 \cdot 8$ & $0 \cdot 8,4 \cdot 1$ & $1 \cdot 2$ & $0 \cdot 6,2 \cdot 3$ \\
\hline Western & $0 \cdot 4$ & $0 \cdot 2,1 \cdot 0$ & $1 \cdot 0$ & $0 \cdot 3,2 \cdot 9$ & $1 \cdot 7$ & $0 \cdot 7,3 \cdot 8$ & 0.6 & $0 \cdot 3,1 \cdot 3$ \\
\hline Nairobi (ref.) & $1 \cdot 0$ & & $1 \cdot 0$ & & $1 \cdot 0$ & & $1 \cdot 0$ & $1 \cdot 0$ \\
\hline \multicolumn{9}{|l|}{ Wealth index } \\
\hline Poorest & 1.5 & $0 \cdot 8,2 \cdot 7$ & $1 \cdot 6$ & $0 \cdot 8,3 \cdot 1$ & $1 \cdot 6$ & $0 \cdot 9,2 \cdot 7$ & $1 \cdot 6$ & $0 \cdot 9,3 \cdot 1$ \\
\hline Poorer & $1 \cdot 6$ & $0.9,2 \cdot 7$ & $1 \cdot 2$ & $0 \cdot 7,2 \cdot 1$ & $1 \cdot 2$ & $0 \cdot 7,2 \cdot 1$ & $1 \cdot 3$ & $0 \cdot 7,2 \cdot 3$ \\
\hline Middle & $1 \cdot 3$ & $0 \cdot 7,2 \cdot 2$ & $1 \cdot \overline{0}$ & $0.5,1.9$ & $1 \cdot 2$ & $0.7,1.9$ & $1 \cdot 3$ & $0 \cdot 8,2 \cdot 2$ \\
\hline Richer & $1 \cdot 3$ & $0 \cdot 8,2 \cdot 2$ & $1 \cdot 1$ & $0.6,1.9$ & $1 \cdot 2$ & $0.7,1.9$ & 0.9 & $0.6,1.5$ \\
\hline Richest (ref.) & $1 \cdot 0$ & & $1 \cdot 0$ & & $1 \cdot 0$ & & $1 \cdot 0$ & \\
\hline Source of drinking water & & & & & & & & \\
\hline Non improved & $1 \cdot 1$ & $0 \cdot 7,1 \cdot 6$ & $0 \cdot 8$ & $0 \cdot 6,1 \cdot 2$ & $1 \cdot 2$ & $0.9,1.5$ & $1 \cdot 1$ & $0 \cdot 8,1 \cdot 4$ \\
\hline Improved (ref.) & $1 \cdot 0$ & & $1 \cdot 0$ & & $1 \cdot 0$ & & $1 \cdot 0$ & \\
\hline Toilet facilities & & & & & & & & \\
\hline No toilet facility & $1 \cdot 2$ & $0 \cdot 7,2 \cdot 3$ & $2 \cdot 7^{\star *}$ & $1 \cdot 5,5 \cdot 0$ & $1 \cdot 1$ & $0 \cdot 7,1 \cdot 8$ & 1.5 & $1 \cdot 2, \cdot 2 \cdot 0$ \\
\hline Non-improved & $1 \cdot 0$ & $0 \cdot 6,1 \cdot 8$ & $2 \cdot 1^{\star *}$ & $1 \cdot 2,3 \cdot 6$ & $1 \cdot 1$ & $0 \cdot 7,1 \cdot 6$ & $1 \cdot 2$ & $0 \cdot 9,1 \cdot 7$ \\
\hline Improved (ref.) & $1 \cdot 0$ & & $1 \cdot 0$ & & $1 \cdot 0$ & & $1 \cdot 0$ & \\
\hline Maternal characteristics & & & & & & & & \\
\hline Mother's age (years) & & & & & & & & \\
\hline$<20$ & $2 \cdot 0^{*}$ & $1 \cdot 1,3 \cdot 7$ & $0 \cdot 8$ & $0.5,1.5$ & 1.5 & $0.9,2 \cdot 6$ & 0.8 & $0 \cdot 5,1 \cdot 3$ \\
\hline $20-30$ years & $1 \cdot 3$ & $1 \cdot 0,1 \cdot 7$ & $0 \cdot 8$ & $0 \cdot 6,1 \cdot 1$ & $1 \cdot 0$ & $0 \cdot 8,1 \cdot 4$ & $0 \cdot 7^{\star}$ & $0 \cdot 6,1 \cdot 0$ \\
\hline$>30$ (ref.) & $1 \cdot 0$ & & $1 \cdot 0$ & & $1 \cdot 0$ & & $1 \cdot 0$ & \\
\hline Education & & & & & & & & \\
\hline No education & $2 \cdot 1^{\star \star}$ & $1 \cdot 3,3 \cdot 3$ & $2 \cdot 5^{\star \star \star}$ & $1 \cdot 5,4 \cdot 0$ & $2 \cdot 7^{\star \star \star}$ & $1 \cdot 8,4 \cdot 0$ & 1.5 & $0.9,2 \cdot 5$ \\
\hline Primary & $1 \cdot 6^{\star \star}$ & $1 \cdot 1,2 \cdot 2$ & $1 \cdot 6^{\star}$ & $1 \cdot 1,2 \cdot 3$ & $1 \cdot 6^{\star \star}$ & $1 \cdot 2,2 \cdot 2$ & $1 \cdot 7^{*}$ & $1 \cdot 2,2 \cdot 4$ \\
\hline Secondary + (ref.) & 1.0 & & $1 \cdot 0$ & & $1 \cdot 0$ & & $1 \cdot 0$ & \\
\hline BMI category & & & & & & & & \\
\hline Pregnant and postpartum & $1 \cdot 1$ & $0.8,1 \cdot 6$ & $1 \cdot 2$ & $0 \cdot 8,1 \cdot 8$ & 0.9 & $0 \cdot 6,1 \cdot 3$ & $1 \cdot 2$ & $0 \cdot 9,1 \cdot 6$ \\
\hline Thin $\left(<18.5 \mathrm{~kg} / \mathrm{m}^{2}\right)$ & $1 \cdot 8^{\star \star}$ & $1 \cdot 3,2 \cdot 5$ & $1 \cdot 8^{\star \star}$ & $1 \cdot 3,2 \cdot 5$ & $1 \cdot 8^{\star \star \star}$ & $1 \cdot 3,2 \cdot 3$ & $1 \cdot 7^{\star \star}$ & $1 \cdot 2,2 \cdot 3$ \\
\hline Overweight/obese $\left(\geq 25.0 \mathrm{~kg} / \mathrm{m}^{2}\right)$ & $0 \cdot 6^{*}$ & $0.4,0.9$ & 0.8 & $0.5,1 \cdot 2$ & $0 \cdot 4^{\star \star *}$ & $0.3,0.7$ & $0.5^{\star \star}$ & $0 \cdot 3,0 \cdot 8$ \\
\hline Normal $\left(18.5-24.9 \mathrm{~kg} / \mathrm{m}^{2}\right.$ ) (ref.) & 1.0 & & 1.0 & & 1.0 & & 1.0 & \\
\hline Number of children $<5$ years old livi & & & & & & & & \\
\hline 1 & $0.5^{\star \star}$ & $0.4,0.8$ & $0 \cdot 6^{\star *}$ & $0.4,0.9$ & $0.5^{\star \star \star}$ & $0 \cdot 4,0 \cdot 7$ & $0 \cdot 6^{\star *}$ & $0.5,0.9$ \\
\hline 2 & 0.8 & $0 \cdot 6,1 \cdot 1$ & $0 \cdot 7^{*}$ & $0 \cdot 6,1 \cdot 0$ & $0 \cdot 7^{\star}$ & $0 \cdot 6,1 \cdot 0$ & $1 \cdot 0$ & $0 \cdot 7,1 \cdot 3$ \\
\hline$\geq 3$ (ref.) & $1 \cdot 0$ & & $1 \cdot 0$ & & $1 \cdot 0$ & & $1 \cdot 0$ & \\
\hline Child-related characteristics & & & & & & & & \\
\hline Sex & & & & & & & & \\
\hline Male & $1 \cdot 5^{\star \star}$ & $1 \cdot 2,1 \cdot 9$ & $1 \cdot 5^{\star *}$ & $1 \cdot 2,1 \cdot 9$ & $1 \cdot 7^{\star \star \star}$ & $1 \cdot 4,2 \cdot 2$ & $1 \cdot 2$ & $1,1 \cdot 5$ \\
\hline Female (ref.) & 1.0 & & $1 \cdot 0$ & & 1.0 & & $1 \cdot 0$ & \\
\hline Age (months) & & & & & & & & \\
\hline$<6$ & $0 \cdot 3^{\star \star \star}$ & $0.2,0.6$ & $0 \cdot 3^{\star \star *}$ & $0.2,0.4$ & $0 \cdot 3^{\star \star \star}$ & $0 \cdot 2,0 \cdot 4$ & $0 \cdot 2^{\star \star *}$ & $0 \cdot 1,0 \cdot 4$ \\
\hline $7-12$ & $0 \cdot 6^{*}$ & $0.4,0.9$ & $0 \cdot 6^{*}$ & $0.4,0.9$ & $0.5^{\star \star \star}$ & $0 \cdot 4,0.7$ & $0 \cdot \overline{7}$ & $0 \cdot 4,1 \cdot 0$ \\
\hline $13-23$ & $1 \cdot 0$ & $0 \cdot 7,1 \cdot 4$ & $0 \cdot 8^{*}$ & $0 \cdot 6,1 \cdot 0$ & 0.9 & $0 \cdot 7,1 \cdot 3$ & $0 \cdot 6^{*}$ & $0.5,0.9$ \\
\hline $24-35$ (ref.) & $1 \cdot 0$ & & $1 \cdot 0$ & & $1 \cdot 0$ & & $1 \cdot 0$ & \\
\hline $36-59$ & 0.8 & $0 \cdot 6,1 \cdot 2$ & $1 \cdot 1$ & $0.9,1.4$ & 0.9 & $0 \cdot 7,1 \cdot 2$ & 0.9 & $0 \cdot 6,1 \cdot 1$ \\
\hline Birth order & & & & & & & & \\
\hline 1 & $0.5^{\star \star}$ & $0.3,0.8$ & $1 \cdot 0$ & $0.6,1.6$ & $0 \cdot 8$ & $0 \cdot 5,1 \cdot 2$ & $1 \cdot 0$ & $0.7,1.5$ \\
\hline 2 or 3 & $0 \cdot 7^{\star}$ & $0.5,1.0$ & $1 \cdot 1$ & $0.8,1.5$ & 0.8 & $0 \cdot 6,1$ & 0.8 & $0 \cdot 6,1 \cdot 0$ \\
\hline$\geq 4$ (ref.) & $1 \cdot 0$ & & $1 \cdot 0$ & & $1 \cdot 0$ & & $1 \cdot 0$ & \\
\hline Size at birth & & & & & & & & \\
\hline Small & $2 \cdot 4^{\star \star \star}$ & $1 \cdot 7,3 \cdot 4$ & $2 \cdot 8^{\star \star \star}$ & $1 \cdot 9,4 \cdot 1$ & $3 \cdot 7^{\star \star \star}$ & $2 \cdot 7,5 \cdot 0$ & $3 \cdot 3^{\star \star \star}$ & $2 \cdot 4,4 \cdot 7$ \\
\hline Average & $1 \cdot 1$ & $0.8,1.5$ & $1 \cdot 4^{*}$ & $1 \cdot 0,1 \cdot 9$ & $1 \cdot 7^{\star \star \star}$ & $1 \cdot 3,2 \cdot 3$ & $1 \cdot 4^{*}$ & $1 \cdot 1,1 \cdot 8$ \\
\hline Large (ref.) & 1.0 & & 1.0 & & 1.0 & & 1.0 & \\
\hline Diarrhoea & & & & & & & & \\
\hline Yes & $0.6^{\star \star}$ & $0.5,0.8$ & $1 \cdot 4^{*}$ & $1 \cdot 0,1 \cdot 9$ & $1 \cdot 7^{\star \star \star}$ & $1 \cdot 3,2 \cdot 2$ & $1 \cdot 1$ & $0.9,1.5$ \\
\hline No (ref.) & 1.0 & & 1.0 & & 1.0 & & $1 \cdot 0$ & \\
\hline
\end{tabular}

ref., reference category.

Note: table is based on results of adjusted binary logistic regression model.

${ }^{\star} P<0.05,{ }^{\star \star} P<0.005,{ }^{\star \star *} P<0.001$.

+1993 and 1998 surveys excluded North Eastern Province. 
country, which has declined from $17 \cdot 4 \%$ in 1993 to $12.7 \%$ in $2008-2009$. Attaining primary level of education is not adequate to give mothers higher human and capital development that in turn would translate into increased family incomes and improved child nutrition.

Maternal undernutrition is a persistent determinant of child undernutrition as seen in our study. Children whose mothers were thin (BMI $<18 \cdot 5 \mathrm{~kg} / \mathrm{m}^{2}$ ) were more likely to be undernourished. Although the study did not investigate the effect of maternal BMI during pregnancy on children's nutrition outcomes, mothers' compromised current nutritional status was shown to be associated with increasing likelihood of undernutrition among the children. The analysis reveals a changing effect of maternal BMI on chronic undernutrition in children, whereby children with overweight mothers were stunted in the 2008-2009 survey. This suggests an emerging pattern of a dual burden of malnutrition, where, in the same household, the mother is overweight while the child is suffering from long-term nutrition deprivation. This coexistence of maternal overnutrition and child undernutrition in the same household describes the rapid nutrition transition in developing countries ${ }^{(24)}$ and has been reported in such middle-income countries as Indonesia and Russia where between $9 \%$ and $13 \%$ of households contain both underweight and overweight members ${ }^{(25)}$.

\section{Conclusions}

The present study set out to analyse the trends and determinants of malnutrition among children $<5$ years of age in Kenya. The study found that the levels of stunting and underweight are decreasing slowly, with more likelihood of undernutrition among children from households in the poorest wealth index, those living in the Coast, North Eastern and Eastern provinces and those who are of small size at birth. The trends in effects of wealth index, maternal education and maternal BMI on child undernutrition suggest shifting patterns with a reduced effect of primary education of mothers and an emerging pattern of the double burden of malnutrition in the same households.

\section{Policy implications}

Considering these important findings, it is critical to translate the national strategies on nutrition interventions in favour of the most vulnerable people in the poorest households as well as those in the most affected regions. One of the goals of the national food and nutrition strategy $2008^{(19)}$ is to increase households' resource productivity. This goal and strategy are examples of a plan that can be targeted to the poorest households with a view to improve the nutritional status of the children. The Kenya government should also take heed of the evidence of changes in maternal education as this has very critical implications for reducing maternal and child malnutrition. Improving maternal education to beyond primary level will make a significant improvement to the nutritional status of children. In order to realize the benefit of improved schooling in a timely manner, education policy makers need to urgently explore methods of ensuring that women of reproductive age who did not have chance to attend secondary schooling have an opportunity to get an equivalent of secondary and higher levels of education through restructuring the education system to allow for adult-based education in the country. Further research to explore the regional disparities of the trends within the country as well as how Kenya compares with other countries in the region is recommended.

\section{Acknowledgements}

This research was made possible by a fellowship funded by the US Agency for International Development (USAID) through the MEASURE DHS project at ICF International. The study research protocol and use of DHS data were approved by ICF International. There are no conflicts of interest. The authors' contributions are as follows: P.K.M., developing research protocol, background information, objectives, data analysis, developing tables and figures, writing up the discussion, recommendations, referencing; D.M., methods, subjects, identifying variables in the DHS databases. The authors would like to thank ICF International for making the data available and the staff team for their support and guidance in statistical analysis of the DHS data.

\section{References}

1. De Onis M, Blössner M, Borghi E et al. (2004) Methodology for estimating regional and global trends of child malnutrition. Int J Epidemiol 33, 1260-1270.

2. Caulfield LE, de Onis M, Blössner M et al. (2004) Undernutrition as an underlying cause of child deaths associated with diarrhea, pneumonia, malaria, and measles. Am J Clin Nutr 80, 193-198.

3. Pelletier DL \& Frongillo EA (2003) Changes in child survival are strongly associated with changes in malnutrition in developing countries. J Nutr 133, 107-119.

4. Grantham-McGregor S, Cheung YB, Cueto S et al. (2007) Developmental potential in the first 5 years for children in developing countries. Lancet 369, 60-70.

5. Ezzati M, Lopez AD, Rodgers A et al. (2002) Selected major risk factors and global and regional burden of disease. Lancet 360, 1347-1360.

6. Chopra M \& Darnton-Hill I (2006) Responding to the crisis in sub-Saharan Africa: the role of nutrition. Public Health Nutr 9, 544-550.

7. Todd B \& Meera S (editors) (2006) Trends and Issues in Child Undernutrition, 2nd ed. Washington, DC: World Bank.

8. Kasirye I (2010) Human Development Research Paper 2010/15: What are the Successful Strategies for Reducing Malnutrition among Young Children in East Africa? New York: UNDP, available at http://hdr.undp.org/es/informes/ mundial/idh2010/trabajos/HDRP_2010_15.pdf 
9. Bryce J, Coitinho D, Darnton-Hill I et al. (2008) Maternal and child undernutrition: effective action at national level. Lancet 371, 510-526.

10. Kabubo-Mariara J, Ndenge GK \& Mwabu DK (2009) Determinants of children's nutritional status in Kenya: evidence from Demographic and Health Surveys. $J$ Afr Econ 18, 363-387.

11. Gewa CA \& Yandell N (2011) Undernutrition among Kenyan children: contribution of child, maternal and household factors. Public Health Nutr (Epublication ahead of print version).

12. ORC Macro (2002) Interviewer's Manual for Use with Model "A" Questionnaire for High Contraceptive Prevalence Countries. MEASURE DHS + Basic Documentation no. 3. http://www.measuredhs.com/pubs/pdf/MBD3/00Front Matter.pdf (accessed March 2012).

13. World Health Organization (2010) WHO Anthro for Personal Computers. Software for Assessing Growth and Development of the World's Children. Geneva: WHO; available at http://www.who.int/childgrowth/software/en/

14. Rutstein S \& Johnson K (2004) The DHS wealth index. DHS Comparative Reports no. 6. http://www.measuredhs. com/publications/publication-CR6-Comparative-Reports.cfm (accessed March 2012).

15. Kenya National Bureau of Statistics \& ICF Macro (2010) Kenya Demographic and Health Survey 2008-09. Calverton, MD: KNBS \& ICF Macro.

16. Pathak PK \& Singh A (2011) Trends in malnutrition among children in India: growing inequalities across different economic groups. Soc Sci Med 73, 576-585.
17. Das S, Hossain MZ \& Nesa MK (2009) Levels and trends in child malnutrition in Bangladesh. Asia Pac Popul J 24, 51-78.

18. Azzarri C, Carletto G, Davis B et al. (2011). Child undernutrition in Mozambique. http://www.protectionblog.org/ wp-content/uploads/2011/12/UNICEF_World-Bank_CarloAzzarri_Child-Undernutrition-in-Mozambique_April-2011. doc protectionblog.org (accessed March 2012).

19. Ministry of Health Kenya (1999) The National Health Sector Strategic Plan 1999-2004. Nairobi: MOH.

20. Getaneh T, Assefa TA \& Taddesse Z (1998) Protein energy malnutrition in urban children: prevalence and determinants. Eth Med J 36, 153-166.

21. Waggoner KM, Jensen E (2011) Evaluating the impact of water and sanitation quality on child malnutrition in SubSaharan Africa. Thesis, Georgetown University.

22. United Nations Development Program (2006) 5th Kenya National Human Development Report. Human Security and Human Development: A Deliberate Choice. Nairobi: UNDP; available at http://mirror.undp.org/kenya/06NHDRreport.pdf

23. Giroux SC (2008) Child Stunting Across Schooling and Fertility Transitions: Evidence from Sub-Saharan Africa. Demographic and Health Working Papers no. 57. Calverton, MD: Macro International Inc; available at http://www. measuredhs.com/pubs/pdf/WP57/WP57.pdf

24. Bouzitou GDN, Fayomi B \& Delisle H (2005) Child malnutrition and maternal overweight in same households in poor urban areas of Benin. Sante 15, 263-270.

25. Doak CM, Adair LS, Bentley M et al. (2005) The dual burden household and the nutrition transition paradox. Int J Obes (Lond) 29, 129-136. 